\title{
A MULTIPHYSICS SIMULATION OF A CORONARY ARTERY
}

\author{
A Thesis \\ presented to \\ the Faculty of California Polytechnic State University, \\ San Luis Obispo
}

\author{
In Partial Fulfillment \\ of the Requirements for the Degree \\ Master of Science in Biomedical Engineering
}

by

Garry Leonard Howe

June 2013 
(C) 2013

Garry Leonard Howe ALL RIGHTS RESERVED 
COMMITTEE MEMBERSHIP

TITLE:

\section{A MULTIPHYSICS SIMULATION OF A CORONARY ARTERY}

AUTHOR:

Garry Leonard Howe

DATE SUBMITTED:

June 2013

COMMITTEE CHAIR:

David Clague, Ph.D., Associate Professor, Department of Biomedical \& General Engineering

COMMITEE MEMBER: $\quad$ Richard Savage, Ph.D., Materials Engineering Department Chair \& Director of Graduate Education

COMMITEE MEMBER: $\quad$ Robert Szlavik, Ph.D., Associate Professor, Department of Biomedical \& General Engineering 


\begin{abstract}
A MULTIPHYSICS SIMULATION OF A CORONARY ARTERY

Garry Leonard Howe
\end{abstract}

This thesis hypothesizes that a commercially available multiphysics simulation software package can be easily employed to characterize the fluid flow and mechanical behavior in healthy and diseased arteries. To this end, multiphysics model of a coronary artery the LAD artery—was created in COMSOL Multiphysics ${ }^{\circledR}$. Wall shear stresses and distension were examined to validate the model. Such a model can be adapted to guide the design of cardiovascular devices. 


\section{TABLE OF CONTENTS}

Page

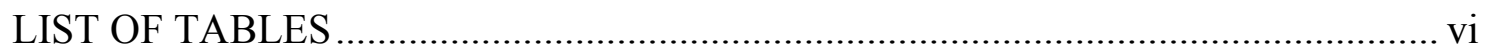

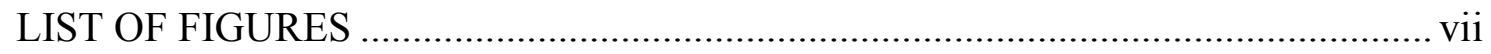

\section{CHAPTER}

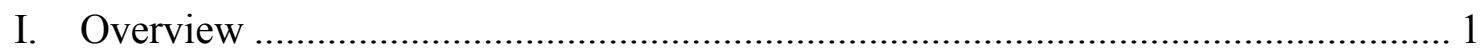

II. Function and Constitution of Arteries ............................................................... 3

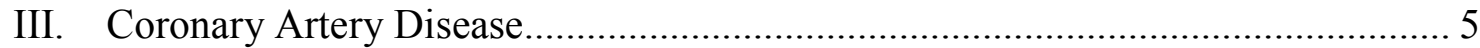

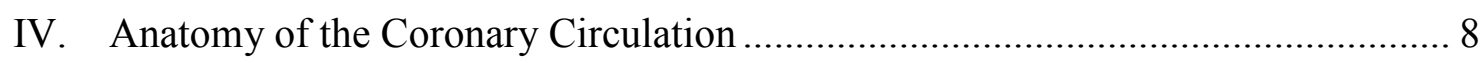

V. Methods for Characterizing Coronary Arteries ..................................................... 11

VI. Physiological Loading Conditions................................................................. 14

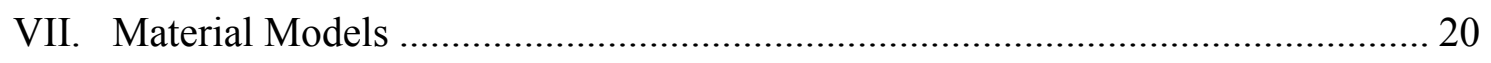

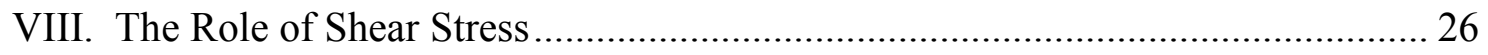

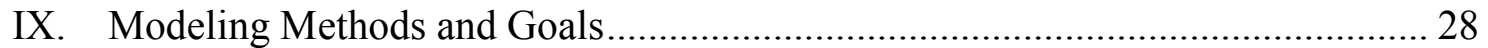

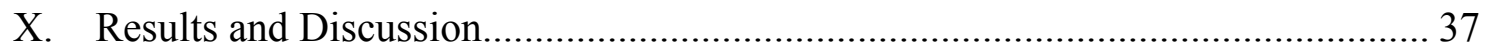

\section{SECTION}

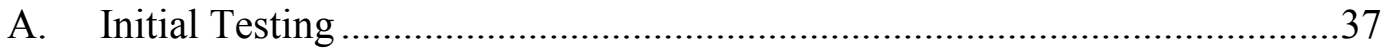

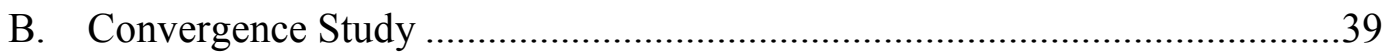

C. Laminar Flow Versus Creep Flow Computations.............................................42

D. Linear Elastic Versus Neo-Hookean Material Models ...................................46

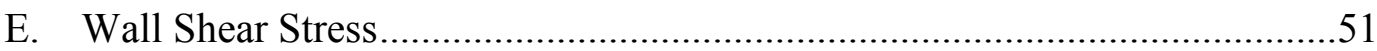

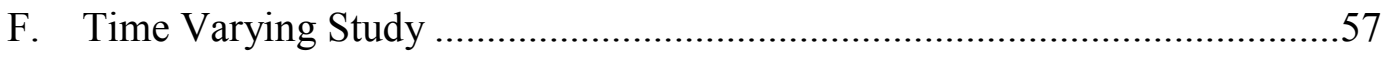

G. Summary and Discussion of Results.........................................................67

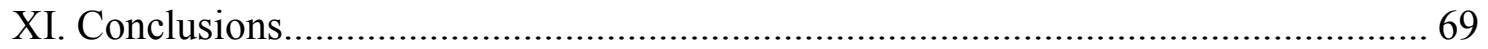

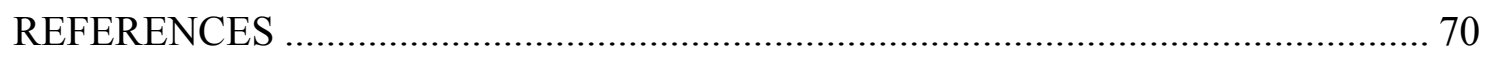




\section{LIST OF TABLES}

Table

1. Average flow rate assigned to each model and associated pressure drop across the artery

2. Comparison of analytical and numerical predictions of several different quantities in the simplified, non-tapered model of a healthy coronary artery.

3. Distension and area change between systole and diastole in the nontapered healthy artery model using an isotropic linear elastic material with varied shear modulus

4. Distension and area change between systole and diastole in the nontapered healthy artery model using an isotropic neo-Hookean elastic material with varied shear modulus.

5. Convergence of several quantities in each tapered artery model using linear elasticity and creep fluid flow.

6. Convergence of several displacement quantities in each tapered artery model using neo-Hookean materials and creep fluid flow

7. Convergence of several fluid dynamic quantities in each tapered artery model using linearly elastic materials and laminar fluid flow. 


\section{LIST OF FIGURES}

Figure Page

1. Layers and morphological features of a typical artery.......................... 4

2. Anterior view of the major arteries of the heart...................................... 9

3. An electrical circuit equivalent to the four element Windkessel model

4. A pressure waveform determined by the 2-element Windkessel model for normal systemic artery conditions.

5. A pressure waveform determined by the 3-element Windkessel model for normal systemic artery conditions.

6. A pressure waveform determined by the 4-element Windkessel model approximating normal systemic artery conditions.

7. Trimetric projection of the tapered geometry used to create the lumen for the healthy artery model

8. Trimetric projection of the tapered geometry used to create the artery wall for the healthy artery model

9. Two-dimension view of geometry revolved to create diseased artery model for (a) a $25 \%$, (b) $50 \%$, and (c) $75 \%$ reduction of lumen radius. (Red-Lumen, Yellow-Plaque, Green-Artery Wall)

10. Comparison of predictions for wall shear stress using "Creep Flow" and "Laminar Flow" physics modules in a model of a healthy tapered coronary artery

11. Comparison of predictions for wall shear stress using "Creep Flow" and "Laminar Flow" physics modules in a model of a tapered coronary artery with a $25 \%$ reduction in lumen radius.

12. Comparison of predictions for wall shear stress using "Creep Flow" and "Laminar Flow" physics modules in a model of a tapered coronary artery with a $50 \%$ reduction in lumen radius....

13. Comparison of predictions for wall shear stress using "Creep Flow" and "Laminar Flow" physics modules in a model of a tapered coronary artery with a $75 \%$ reduction in lumen radius 
14. Comparison of lumen area change between a healthy tapered artery modeled with an isotropic linearly elastic material and an isotropic neo-Hookean material model.

15. Comparison of radial stress distribution between a healthy tapered artery modeled with an isotropic linearly elastic material and an isotropic neo-Hookean material model

16. Comparison of radial stress distribution between a tapered artery with a $25 \%$ reduction in lumen radius modeled with an isotropic linearly elastic material and an isotropic neo-Hookean material model

17. Comparison of radial stress distribution between a tapered artery with a 50\% reduction in lumen radius modeled with an isotropic linearly elastic material and an isotropic neo-Hookean material model

18. Comparison of radial stress distribution between a tapered artery with a $75 \%$ reduction in lumen radius modeled with an isotropic linearly elastic material and an isotropic neo-Hookean material model

19. Distribution of wall shear stress in a model of a healthy tapered artery using creeping flow with an average volumetric flow rate

20. Distribution of wall shear stress in a model of a tapered artery with a $25 \%$ reduction in lumen radius using creeping flow with an average volumetric flow rate.

21. Distribution of wall shear stress in a model of a tapered artery with a $50 \%$ reduction in lumen radius using creeping flow with an average volumetric flow rate.

22. Distribution of wall shear stress in a model of a tapered artery with a $75 \%$ reduction in lumen radius using creeping flow with an average volumetric flow rate.

23. Comparison of the distribution of wall shear stress in each model of a tapered artery using creeping flow with an average volumetric flow rate.

24. Points along artery wall tracked over time for each tapered model. 58 
25. Primary axis-Comparison of the displacement of three different points along a healthy artery over one cardiac cycle; Secondary axisSystemic blood pressure applying load.

26. Primary axis-Comparison of the displacement of three different points along an artery with $25 \%$ reduced lumen radius at the center over one cardiac cycle; Secondary axis-Systemic blood pressure applying load.

27. Primary axis-Comparison of the displacement of three different points along an artery with $50 \%$ reduced lumen radius at the center over one cardiac cycle; Secondary axis-Systemic blood pressure applying load.

28. Primary axis-Comparison of the displacement of three different points along an artery with $75 \%$ reduced lumen radius at the center over one cardiac cycle; Secondary axis-Systemic blood pressure applying load.

29. Primary axis-Comparison of the wall shear stress of three different points along a healthy artery over one cardiac cycle; Secondary axisSystemic blood pressure applying load.

30. Primary axis-Comparison of the wall shear stress of three different points along an artery with a $25 \%$ reduced lumen radius over one cardiac cycle; Secondary axis-Systemic blood pressure applying load.

31. Primary axis-Comparison of the wall shear stress of three different points along an artery with a $50 \%$ reduced lumen radius over one cardiac cycle; Secondary axis-Systemic blood pressure applying load.

32. Primary axis-Comparison of the wall shear stress of three different points along an artery with a $75 \%$ reduced lumen radius over one cardiac cycle; Secondary axis-Systemic blood pressure applying load. 


\section{Overview}

Finite Element Analysis (FEA) can be a particularly powerful tool for studying the behavior of many systems, including medical devices and biological tissues. While there are limitations, it can be useful when predicting the performance of a new device design before creating a prototype, an often costly procedure. Although such designs should ultimately be validated physically, knowing of potential issues or improvements in an in-silico model would useful for determining whether or not to it is wise to move forward with a particular design. These insilico models are quite useful for cardiovascular devices [1,2], which continue to have new designs created in an attempt to improve their performance in-vivo [3-5].

In the context of biomedical applications, the use of multiphysics modeling is relatively new; considering solid and fluid mechanical behaviors is important for many problems present in this field of study [6]. Although a single physics approach may be simpler to implement, it provides an incomplete representation of the in-vivo conditions, particularly if the model is for devices and pathologies that are effected by the flow field. A multi-physics approach that incorporates the solid mechanical behavior of the artery as well as the fluid dynamic behavior of the blood passing through it would provide a more accurate prediction of in-vivo behavior. 
Multiphysics simulation software packages, such as COMSOL Multiphysics ${ }^{\circledR}$, are commercially available. This thesis hypothesizes that a multiphysics simulation of both diseased and healthy arteries can be readily implemented in such a software package. To this end, using COMSOL ${ }^{\circledR}$, several multiphysics models of a coronary artery were created. Wall shear stresses and distension were examined to validate the models against literature. Ideally these models can be adapted to incorporate cardiovascular devices to predict their behavior before physical prototypes are created. 


\section{Function and Constitution of Arteries}

Arteries are the blood vessels that carry blood away from the heart, either to supply the lungs with deoxygenated blood or to supply the rest of the body with oxygenated blood. As the distribution of metabolites by diffusion alone has only a very short effective range, the constant perfusion of blood through these vessels is of the utmost importance to life. These vessels constitute the higher pressure portion of the circulatory system; they originate at the aorta and branch into smaller systemic arteries that service the different parts of the body [7]. Branching continues until arterioles are formed, which help to regulate blood pressure by modulating their resistance to fluid flow. Arterioles then separate into capillaries, which are smaller in size than arterioles and are optimized for the exchange of metabolites by diffusion. These small vessels greatly support normal metabolism in the adjacent cells.

Arteries are composed of several layers of tissue as illustrated in Figure 1 [7]. The tunica intima is the innermost layer of an artery; it is comprised of a thin layer of endothelial cells that line the lumen of the vessel. The endothelium is supported by the internal elastic lamina. As blood flows through the lumen, it contacts the endothelium and influences the vessel's physiology. Alterations to the flow or the endothelium can have profound effects leading to vascular pathogenesis, the development of often serious disease. The tunica media surrounds the intima; it is comprised of smooth muscle cells (SMCs) and elastic tissue, the 
proportions of which depend on the particular artery, with the larger arteries typically being more elastic to manage the pulsatile flow of blood. Finally, the outermost layer of an artery is the tunica externa, which is largely composed of collagen. It is supported by the external elastic lamina and helps to secure the artery in place relative to nearby organs and tissues.

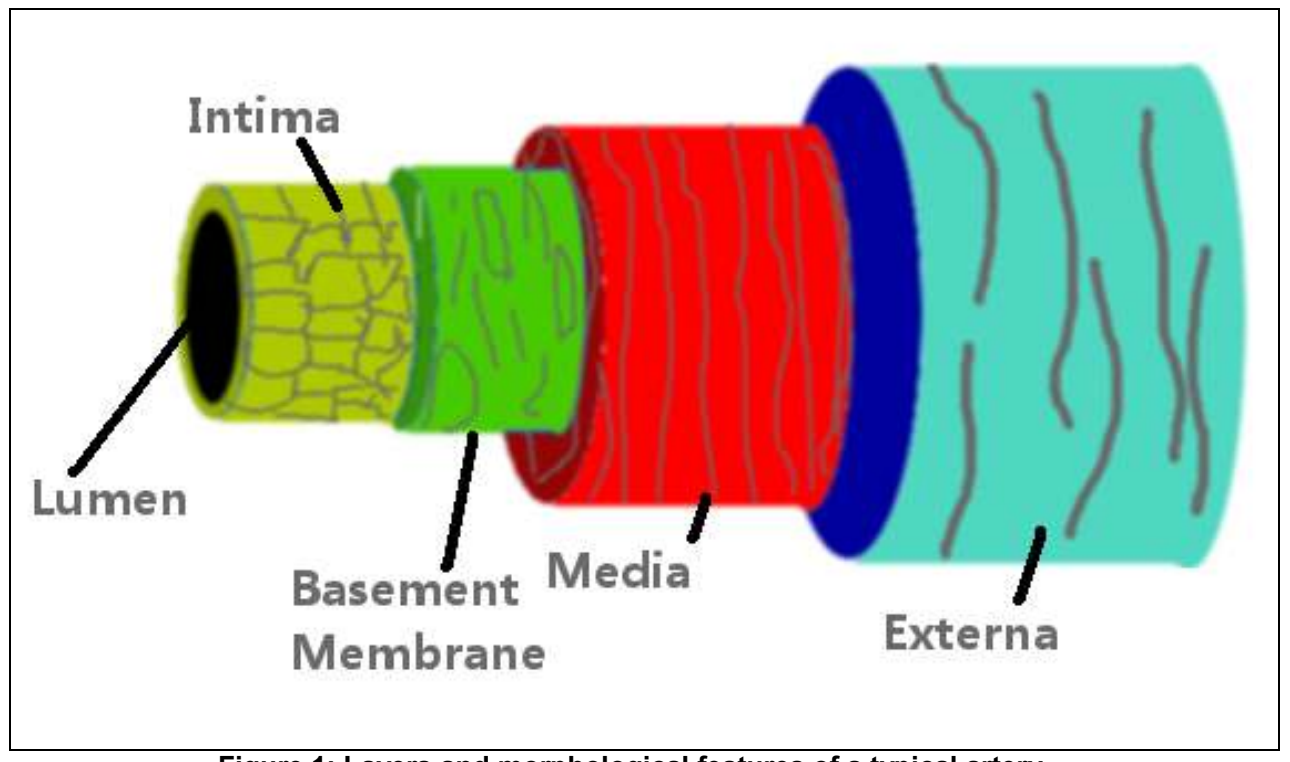

Figure 1: Layers and morphological features of a typical artery. 


\section{Coronary Artery Disease}

Coronary artery disease (CAD) affects about 16.8 million people in the United States [8]. It is the leading cause of death nation-wide [9]. This disease occurs when the arteries supplying the heart muscle blood develop atherosclerotic plaques on their inner walls, resulting in the stiffening of the blood vessels. To compensate for the decreased compliance, the arteries grow wider; however, this response is not perfect and the vessels can ultimately narrow, restricting blood flow and placing an increased burden on the heart. This may begin to cause symptoms such as angina pectoris (chest pain). The effects of ischemia, the decreased flow of blood resulting in decreased efficiency of the heart, may be reversible with treatment while more dramatic symptoms such as myocardial infarction (heart attack) are permanent.

Atherosclerotic lesions begin to form in the tunica intima; there are some secondary changes in the tunica media as well [10]. In the earliest stages, macrophages and smooth muscle cells (SMCs) accumulate in the intima. With time, the SMCs proliferate followed by the production of extra-cellular matrix elements such as collagen and elastin. Finally, lipids accumulate in the cells and the connective tissue. All of this contributes to stiffening and stenosis. One early characteristic of atherosclerosis is the appearance of fatty streaks along the vessel walls due to lipid accumulation by the intimal macrophages and SMCs. These streaks 
generally start appearing during childhood in the aorta and then, later, in other arteries.

Early lesions are flat or only slightly raised; they tend to be long and narrow [10]. As the disease progresses, an atheroma-a fibrous plaque-can begin to form. Ultimately, a mass of lipid becomes encapsulated in the intima and separated from the lumen by a fibrous cap. The lipid content can vary depending on the artery in which the lesion is found. Coronary artery lesions resulting in severe stenosis can exhibit calcification, ulceration, and cell necrosis. These plaques can rupture and cause angina, myocardial infarction, and death. Embolized debris can also induce thrombosis that ultimately causes serious issues elsewhere in the body.

While research continues, there have been several treatments developed to address stenosis caused by atherosclerotic arteries. Percutaneous transluminal coronary angioplasty (PCTA) is a procedure to correct stenosis non-invasively by deploying a balloon via catheter under the guidance of fluoroscopy. This was a breakthrough as it allowed for an alternative to invasive bypass surgeries, which often only warrant the associated high risks in patients with very widespread and severe occlusions throughout the coronary circulation. Until the early 1990s, PCTA alone was quite common and continued to grow in popularity; however, the procedure's major common issue was the high occurrence of restenosis requiring repeated intervention [11]. Given this commonly 
occurring issue, physicians started to adopt stent deployment with PCTA. Currently, this procedure is quite common; according to the Centers for Disease Control, approximately 500,000 coronary stents were implanted in the year 2009 [12]. Although the performance of stents has improved over the years, there is still room for improvement with regards to design and deployment $[4,5]$. For these reasons, in-silico models of an artery would be of high value as a preliminary test of novel techniques and device designs. 


\section{Anatomy of the Coronary Circulation}

To support the crucial function of contracting to pump blood, the heart muscle receives its own blood supply from several arteries that branch off of the aorta. The heart receives blood through the coronary circulation at an average rate of approximately $250 \mathrm{~mL} / \mathrm{min}$, which translates to about $5 \%$ of the total flow output [13]. There are many vessels feeding the oxygenated blood to the heart tissues, as illustrated in

\section{Figure 2.}

Some of the coronary blood flow goes through the right main coronary artery (RMCA), which originates from the aorta and runs down the right atrioventricular groove; it provides blood to the right side of the heart and makes a contribution to the supply for the left side as well [14]. The RMCA features a marginal branch artery (the RMA) that supplies the right ventricle. Most individuals have right side dominance in which the RMCA branches into the posterior descending artery (PDA) that supplies blood to about a third of the septum, though this branch can sometimes originate from the left side coronary arteries in those with left dominance.

The left side of the heart is supplied blood by the left main coronary artery (LMCA), which branches off from the aorta and then runs a short length before bifurcating into the left anterior descending (LAD) artery and the left circumflex artery (LCX) $[14,15]$. Occlusion of the LAD is a particularly grave concern; it supplies blood to a large portion of the anterior and septal regions of the left ventricle, which are responsible 
for much of the force generated to pressurize and propel blood. This artery is quite prone to atherosclerosis and narrowing; for these reasons, an occluded LAD is sometimes called "the widow maker" [15]. The LAD has several smaller branches of its own [16]. Septal branches, which vary considerably in size and number between individuals, take off almost orthogonally to supply the interventricular septum, helping to make this region of the heart one of the most densely vascularized. An average of three or more diagonal branches are often present as well.

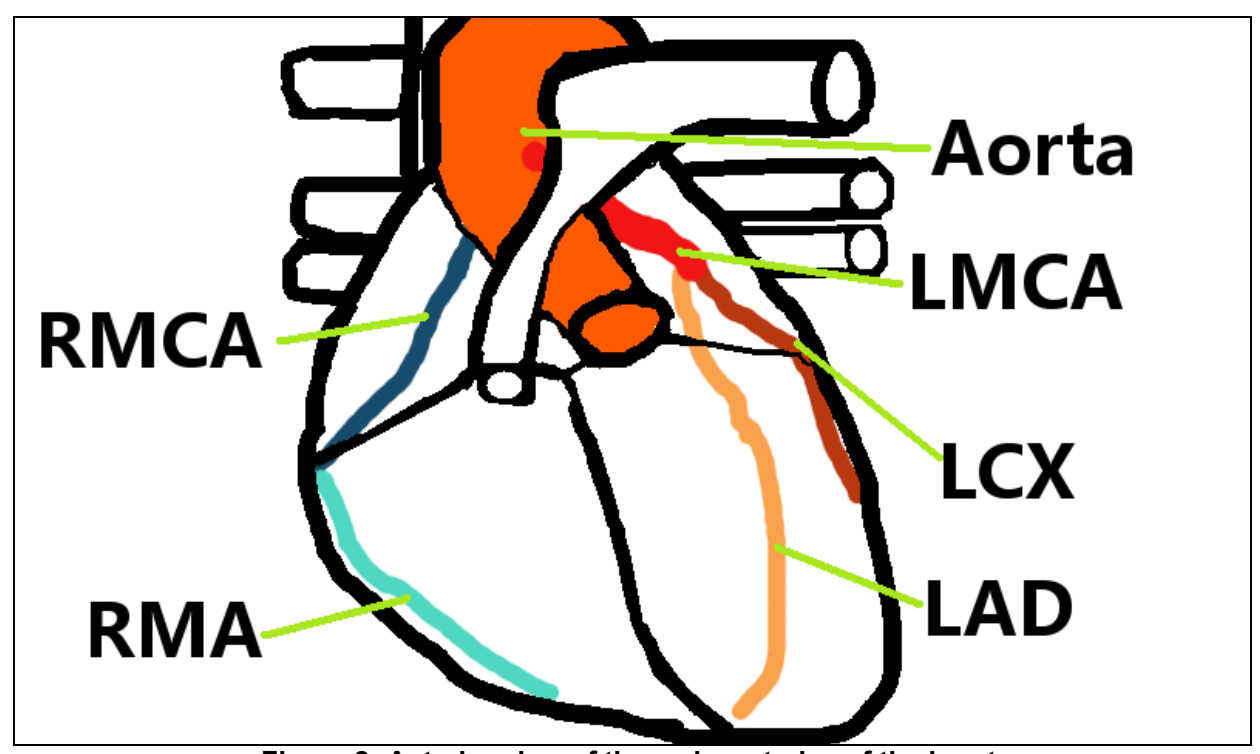

Figure 2: Anterior view of the major arteries of the heart.

Given the significance of the LAD and the readily available data for it, the model arteries were given the dimensions of its anatomy. The total length of this artery is about $6.4 \mathrm{~cm}$ on average [17-19]. In healthy individuals, the artery wall is amount $1 \mathrm{~mm}$ thick [20-23], while people with CAD have thicker walls, often reported as about $1.8 \mathrm{~mm}$ thick in diseased portions $[21,23,24]$. It should be noted though that individual cases may vary considerably. The LAD is tapered, featuring a changing 
lumen area by a little over a square millimeter per centimeter of artery [25]; these vessels are widest at their origin and narrow towards the distal end. Reported values for the lumen diameter vary, ranging from a minimum about $2 \mathrm{~mm}[22,23,26]$ to a maximum of about $3.5 \mathrm{~mm}[24,26$, 27], which, taken as approximations of the distal and proximal ends respectively, roughly correspond to the quantified amount of taper predicted above and creates an average diameter in line with these studies. Lumen diameter differences between healthy subjects and patients with mild CAD are minimal $[23,24]$. Wall thickness and inner diameter do not appear to be significantly different between patients with mild CAD and somewhat more severe cases [28]. Patients with extremely severe cases of CAD have large atherosclerotic lesions built on the inner walls that reduce the effective size of the lumen, leading to ischemia and the associated risks. 


\section{Methods for Characterizing Coronary Arteries}

Numerous techniques exist for collecting the data to build a mathematical model of an artery. Certain imagining techniques can be used to gather in-vivo data; they are particularly useful for determining geometry and blood flow velocities, and can also provide insights into arterial composition. Other techniques can also be used ex-vivo to measure mechanical properties and closely examine layer thickness and histological composition.

Intravascular ultrasound (IVUS) is one of several imaging techniques that can estimate radial strain and material composition in arteries $[29,30]$. With this imagining technique, a piezoelectric transducer is used in a probe that is attached to the distal end of a catheter. This technique can typically provide a resolution of about 70 to $200 \mu \mathrm{m}$ depending on the frequencies used [31]. The time it takes for signals to return to the transducer after reflecting off a surface determines the apparent distance and direction of an object. Differences in acoustic impedance, a function of the speed of sound in the medium as well as its mass density, are responsible for the reflections and amount of energy returned. These differences dictate the brightness of a region in the image. The resultant images are typically analyzed with software. IVUS is also useful for observing characterizing plaques in patients [29, 30].

It is important to note that other ultrasound techniques are also useful. Doppler ultrasound imaging can be used to determine blood 
velocity; combined with a knowledge of the vessel geometry, it is possible to gain insights into in-vivo hemodynamics. The velocity can be determined because a moving object reflecting a wave will cause an apparent change in the frequency of the returning wave.

Another very useful approach in particular for examining soft tissues is Magnetic Resonance Imaging (MRI). In this imaging technique, a strong magnetic field aligns the magnetic moments of the abundant water molecules and hydrogen ions in the body. The electromagnetic field is then altered, driving certain molecules to resonate at certain frequencies. When the field exciting the molecules is removed, they emit radiofrequency electromagnetic waves that are recorded, and, via Computer Tomography methods, are used to construct an anatomical image. The technique can create images with a resolution of about 100 $\mu \mathrm{m}[31]$. Since this technique can easily construct three dimensional images, it can be used to create geometries to mesh for finite element analysis. There have been studies using MRI to analyze plaque composition and geometry that correlate well with histological evaluation [32].

Optical Coherence Tomography (OCT) has also been used to characterize plaques both in-vitro [33] and in-vivo [34]. It is a very high resolution imaging method that uses an interferometric technique with near infrared frequencies of light, typically providing a precision of 10 to 
$20 \mu \mathrm{m}[31] .$. In some respects, the principle of operation for this technique is similar to ultrasound.

Perhaps the simplest and most direct methods for characterizing arteries involve dissection followed by histological and mechanical examination. Samples can be dissected into their constitutive tissue types or may be left whole to develop a simpler homogenous approximation of the mechanical behavior. Mechanical properties are ideally measured multi-axially and over a wide range of strains to develop accurate stressstrain relationships. In addition to human tissue samples, many studies estimate the material properties by testing animal specimens. For example, porcine arteries are studied because of the ease of acquiring and preparing them, as well as for their similarities to human arteries [35]. 


\section{Physiological Loading Conditions}

While measuring coronary arterial blood pressure directly can be very difficult and invasive, it is possible to measure the velocity of blood through particular vessels using noninvasive methods, including Doppler ultrasound and MRI. Velocity data combined with a knowledge of blood vessel radius and length can be used to estimate the pressure drop across the vessel using the Hagen-Poiseuille equation (Equation 1):

$$
Q=\frac{\pi r^{4}}{8 \mu L} \Delta P=\langle v\rangle A
$$

This equation assumes a straight, rigid cylindrical vessel carries a fluid experiencing laminar flow, a reasonable assumption for many blood vessels, though it is an approximation for the larger arteries, which tend to taper, curve, and exhibit compliance. The fluid flow rate $\mathrm{Q}$ is estimated as the product of the lumen area $A$ and the average blood velocity $\langle v\rangle$, and the resistance of the vessel is determined on its length $L$, radius $r$, and the viscosity $\mu$ of the blood flowing through it.

In the $\mathrm{LAD}$, the blood velocity peaks up to about $33 \mathrm{~cm} / \mathrm{s}$ during diastole [36, 37] and averages about $20.5 \mathrm{~cm} / \mathrm{s}[37,38]$. With these figures, for example, assuming a blood viscosity of $5 \mathrm{mPa}-\mathrm{s}$, a cylinder of length $6.4 \mathrm{~cm}$ and radius of $2.75 \mathrm{~mm}$ has a predicted a peak pressure difference of about $450 \mathrm{~Pa}$ and an average difference of $275 \mathrm{~Pa}$.

For greatly occluded arteries, it should be noted that the coronary flow reserve (CFR) is noticeably reduced from about 2.5 , as seen in 
healthier arties, to about $1.5[36,37]$. This ratio of the flow in the hyperemic state to that of the basal state provides insight into how well perfused tissue is; the greatly stenosed arteries deliver noticeably less blood in the hyperemic state than their healthy counterparts and less flow overall.

Several models exist for the systemic blood pressure waveform seen under normal physiological conditions. The Windkessel model, one of the earliest developed, is a simple representation of the cardiovascular system described in Equation 2:

$$
Q_{I n}=\frac{d V}{d t}+Q_{O u t}
$$

In this model, blood flows from the heart at the rate $Q_{\text {in }}$ into the larger arteries of compliance $C$ that have a volume $V$; the blood then leaves at a

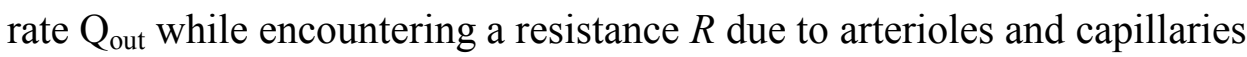
$[39,40]$. This can be rearranged in terms of the systemic pressure $P$ in Equation 3; the resultant relationship is quite similar to an electrical capacitor and resistor in parallel:

$$
Q_{I n}=C \frac{d P}{d t}+\frac{P}{R}
$$

This simplest version of the Windkessel model is referred to as the 2 element model; additional elements are sometimes added to improve the accuracy of the model. In the electrical circuit analogy, it is as though a resistor is added in series to the original pair of elements to create a 3 element model. The four element model adds in an inductance in series as 
seen in Figure 3. These additional terms modify the relationship slightly as seen in Equation 4.

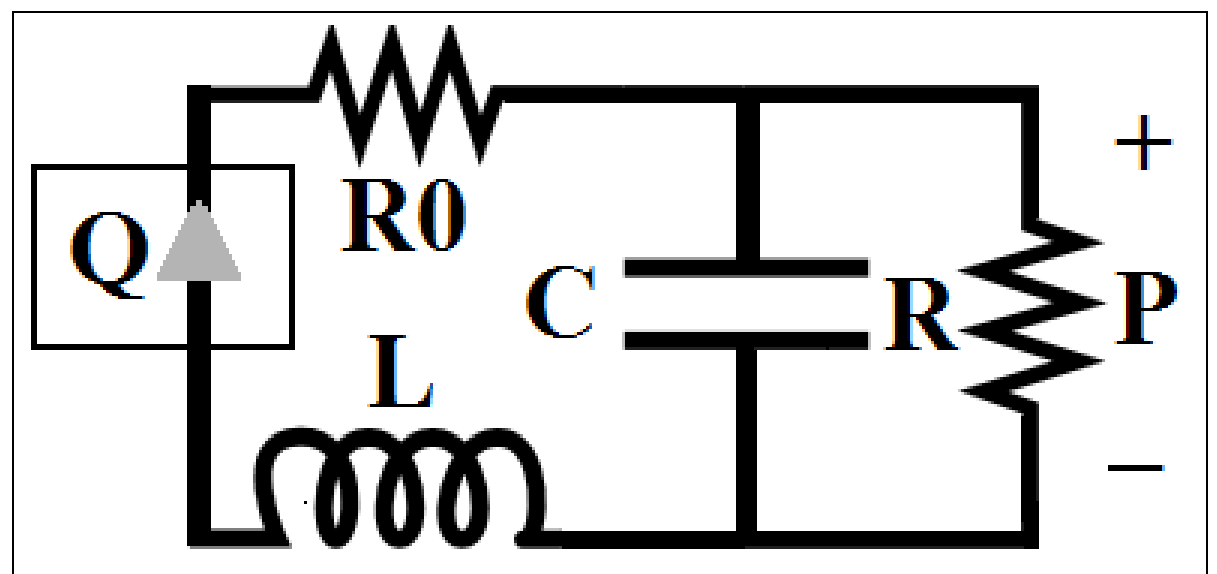

Figure 3: An electrical circuit equivalent to the four element Windkessel model.

$$
\left(1+\frac{R_{0}}{R}\right) Q+\left(C R_{0}+\frac{L}{R}\right) \frac{d Q}{d t}+L C \frac{d^{2} Q}{d t^{2}}=C \frac{d P}{d t}+\frac{P}{R}
$$

During diastole, the model assumes a constant zero inward flow of blood [39], which results in an exact exponential decay function from the value of the pressure at the start of diastole (Equation 5):

$$
P(t)=P\left(t_{D}\right) e^{-\left(t-t_{D}\right) / R C} \quad t_{D} \leq t \leq T
$$

It should be noted that this part of the solution also works for the more complicated 3 and 4 element Windkessel models since there is still no flow during diastole, resulting in the left hand side of the equation being zero; this part of the solution can therefore be incorporated easily into the total solution for each case. 
During systole, the model assumes blood flows, meaning that must $\mathrm{Q}_{\text {in }}$ term must be incorporated. One simple approximation for this term is given by Equation 6:

$$
Q_{i n}(t)=\left\{\begin{array}{cc}
Q_{0} \sin \left(\frac{\pi}{h} \operatorname{rem}\left(\frac{t}{T}\right)\right) & \begin{array}{c}
\frac{t}{T} \leq h \\
0
\end{array} \\
\text { otherwise }
\end{array}\right.
$$

Here, $T$ is the period of the cardiac cycle, rem() indicates the remainder of the division is taken, and $h$ is the time for which the valves of the heart are open [39].

With this fairly simple approximation for the flow rate and by rearranging the governing differential equation to Equation 7, one can easily find a solution numerically in programs such as MATLAB®;

Figures 4 through 6 show example waveforms for the two, three, and four

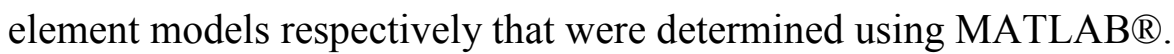
MATLAB ${ }^{\circledR}$ has an advantage compared to other programs in that COMSOL Multiphysics ${ }^{\circledR}$ can evaluate its functions. As an alternative, one may import a load curve as an "Interpolation Function" that reads a spreadsheet of values.

$$
\frac{d P}{d t}=\frac{1}{C}\left[\left(1+\frac{R_{0}}{R}\right) Q+\left(C R_{0}+\frac{L}{R}\right) \frac{d Q}{d t}+L C \frac{d^{2} Q}{d t^{2}}-\frac{P}{R}\right]
$$


Systemic Blood Pressure Over One Cardiac Cycle Predicted by a 2 Element Windkessel Model

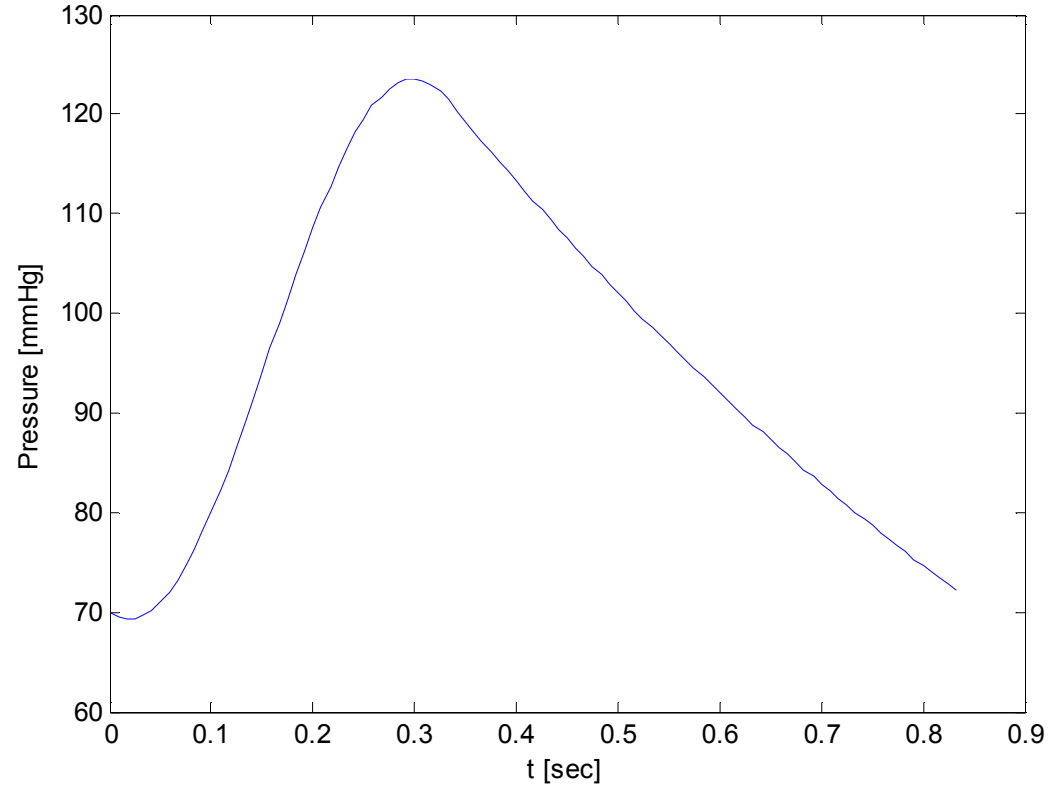

Figure 4: A pressure waveform determined by the 2-element Windkessel model for normal systemic artery conditions.

Systemic Blood Pressure Over One Cardiac Cycle Predicted by a 3 Element Windkessel Model

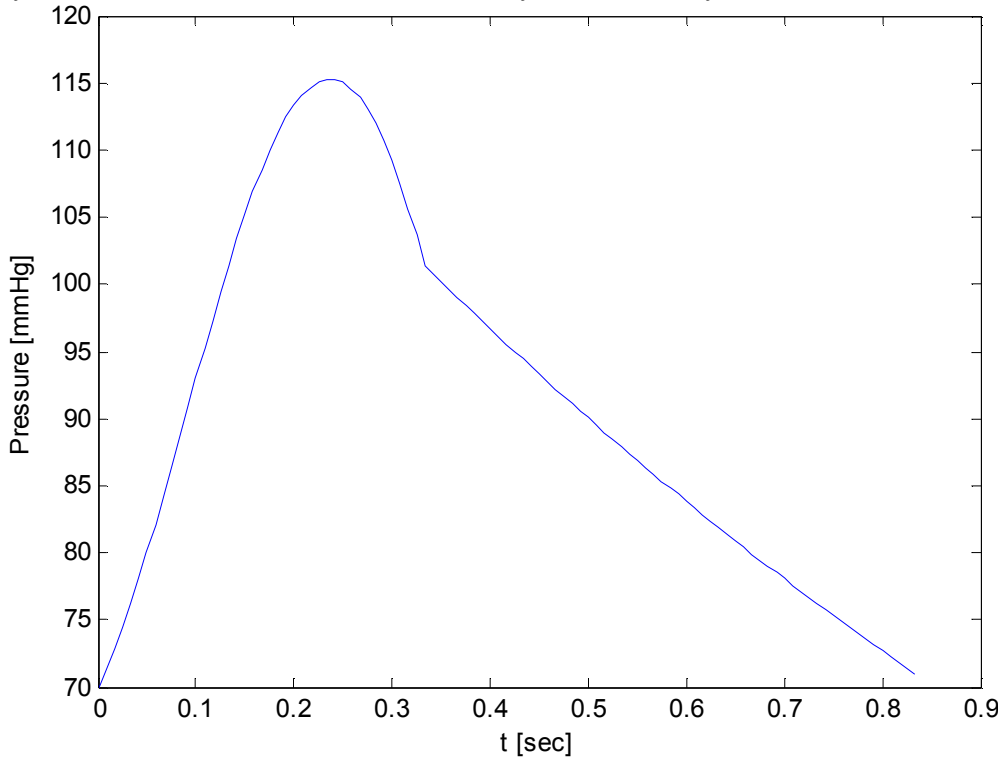

Figure 5: A pressure waveform determined by the 3-element Windkessel model for normal systemic artery conditions. 


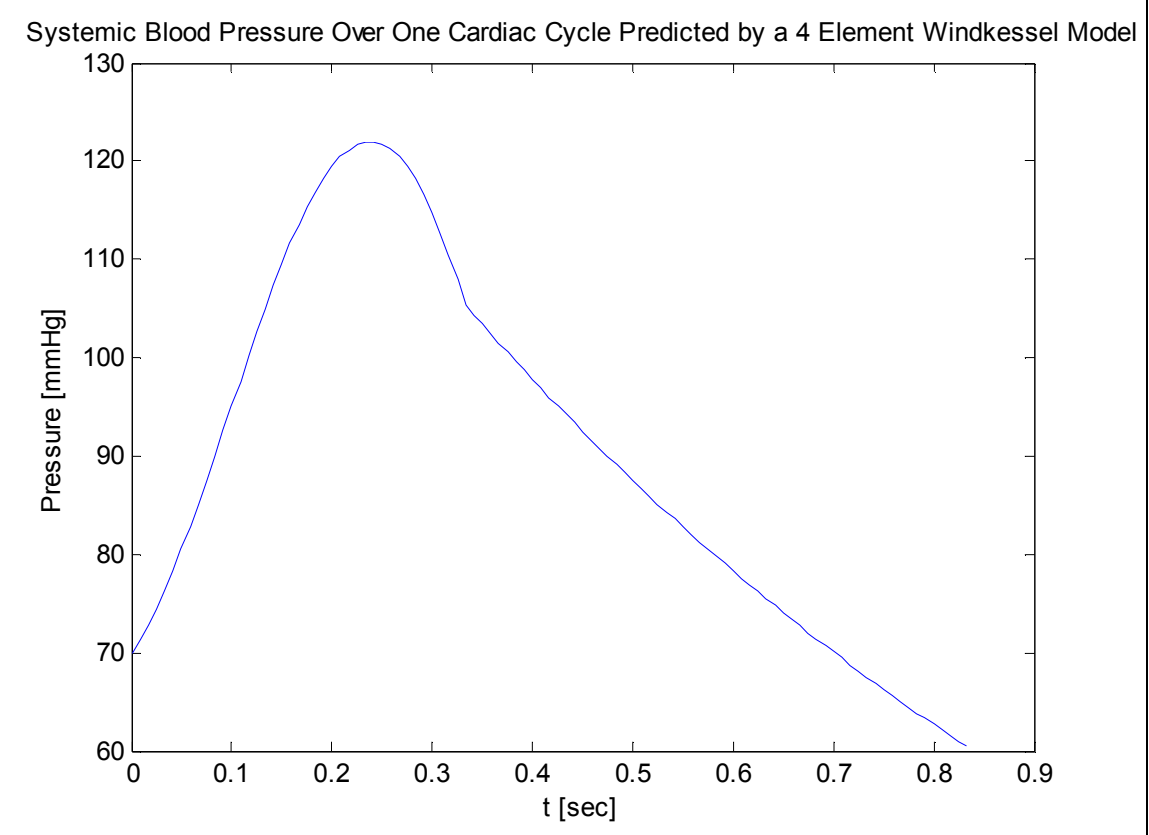

Figure 6: A pressure waveform determined by the 4-element Windkessel model approximating normal systemic artery conditions.

One observation that is incorporated into some artery models is that of arterial residual stress and strain [41]. When arteries are cut open longitudinally, they tend to roll open, indicating that their usual circular cross-sectional configuration is not the zero stress state. Incorporating this consideration into models tends to result in more uniform distributions of stress; the implications are more profound for models incorporating multiple distinct layers of the artery [41]. Each layer tends to separate with a different opening angle into the zero stress state.

In addition to this residual stress, researchers have also found that in-situ arteries are axially stretched [42]. Studies have found LAD segments to be stretched to a ratio of 1.04 in-situ in human specimens and a greater ratio of about 1.35 in the porcine model [43-45]. 


\section{Material Models}

Biological tissues differ from most engineering materials; they typically undergo large strains and exhibit non-linear stress-strain relationships similar to rubbers. Compared to other tissues though, coronary arteries undergo relatively small strains. In such scenarios, a linear material model approximation may be somewhat appropriate. Some studies have found the stress strain relationship to be nearly linear in when evaluated under loads similar to normal physiologic pressures [46]. In the context of finite element analysis, linear relationships allow for a direct solution method without the need for a potentially computationally costly iterative approach. However, it must be noted that a linear approach may not be the most accurate.

Several nonlinear solid material models exist. In these models, a strain energy function $W$ relates the stresses experienced by a material to the deformation as shown in Equations 8 \& 9:

$$
\begin{aligned}
P_{i A} & =\frac{\partial W}{\partial F_{i A}}=2 F_{i B} \frac{\partial W}{\partial C_{A B}} \\
t_{i j} & =2 J^{-1} F_{i A} F_{j B} \frac{\partial W}{\partial C_{A B}}
\end{aligned}
$$

Here, $F_{i A}$ is the deformation gradient tensor, $C_{A B}$ is the right Cauchy-Green deformation tensor, $J$ is the Jacobian of the transformation, $P_{i A}$ is the first Piola-Kirchhoff stress tensor, and $t_{i j}$ is the Cauchy stress tensor.

Often, the stress tensor is expressed in terms of the three invariants of the Cauchy-Green deformation tensor as shown in Equation 10, which, 
by definition, do not vary depending upon the coordinate or reference system used:

$t_{i j}=\left[2 I_{3}^{C_{A B} 1 / 2} \frac{\partial W}{\partial I_{3}^{C_{A B}}}\right] \delta_{i j}+\left[2 I_{3}^{C_{A B}-1 / 2}\left(\frac{\partial W}{\partial I_{1}^{C_{A B}}}+I_{1}^{C_{A B}} \frac{\partial W}{\partial I_{2}^{C_{A B}}}\right)\right] B_{i j}+\left[-2 I_{3}^{C_{A B}}-1 / 2 \frac{\partial W}{\partial I_{2}^{C_{A B}}}\right] B_{i k} B_{k j}$ (10)

Here, $I_{n}$ is the $n$th invariant of the right Cauchy-Green deformation tensor $C_{A B}$ and $\mathrm{B}_{\mathrm{ij}}$ is the left Cauchy-Green deformation tensor.

A commonly used hyper-elastic model material class for arteries is the isotropic Mooney-Rivlin rubber as described by Equation 11; it is also common to use the related subset of materials that follow the neo-Hookean material model shown in Equation 12. In these equations, $C_{1}, C_{2}$, and $D_{1}$ are constants.

$$
\begin{gathered}
W=C_{1}\left(I_{1}^{C_{A B}}-3\right)+C_{2}\left(I_{2}^{C_{A B}}-3\right)+D_{1}(J-1)^{2} \\
W=C_{1}\left(I_{1}^{C_{A B}}-3\right)+D_{1}(J-1)^{2}
\end{gathered}
$$

It is important to note that the third invariant of the Cauchy-Green deformation tensors depends on the determinant of the transformation, which in turn indicates the change in volume of the material associated with that deformation. For incompressible materials, this determinant stays a constant value of one, as does the Jacobian $J$ of the deformation gradient $F$. Since the third invariant does not change, the derivative of the strain energy function with respect to that invariant is zero, simplifying hyperelastic models when this constraint is applied. As artery walls are considered effectively incompressible $[47,48]$, one may simplify the strain energy functions of the isotropic Mooney-Rivlin material model as seen in Equation 13 and Neo-Hookean material model as seen in Equation 14. In the linear elastic material model, this volume preserving constraint 
translates into a Poisson's ratio $v$ of nearly 0.5 , or in other terms, a very large, effectively infinite bulk modulus.

$$
\begin{gathered}
W=C_{1}\left(I_{1}^{C_{A B}}-3\right)+C_{2}\left(I_{2}^{C_{A B}}-3\right) \\
W=C_{1}\left(I_{1}^{C_{A B}}-3\right)
\end{gathered}
$$

In order to be consistent with linear elasticity, the constants of the Mooney-Rivlin strain energy function are related to the shear modulus $G$ as shown below in Equations 15-17, which consider a pure shear deformation of a Mooney-Rivlin material.

$$
\begin{gathered}
F_{i A}=\left[\begin{array}{ccc}
1 & \alpha & 0 \\
0 & 1 & 0 \\
0 & 0 & 1
\end{array}\right] \rightarrow B_{i j}=F F^{T}=\left[\begin{array}{ccc}
1+\alpha^{2} & \alpha & 0 \\
\alpha & 1 & 0 \\
0 & 0 & 1
\end{array}\right] \\
t_{i j}=-p \delta_{i j}+\left[\begin{array}{ccc}
2\left(C_{1}-C_{2}\right)+2 C_{1} \alpha^{2} & 2\left(C_{1}+C_{2}\right) \alpha & 0 \\
2\left(C_{1}+C_{2}\right) \alpha & 2\left(C_{1}-C_{2}\right)-2 C_{2} \alpha^{2} & 0 \\
0 & 0 & 2\left(C_{1}-C_{2}\right)
\end{array}\right]
\end{gathered}
$$

Here, Equation 15 shows the deformation gradient $F_{i A}$ associated with pure torsion, the amount of which is determined by the scalar $\alpha$. The resultant left Cauchy-Green deformation tensor $B_{i j}$ is also shown. These terms are substituted along with those associated with the incompressible MooneyRivlin strain energy function (Equation 13) into the expression for Cauchy stress given in Equation 10. The resultant stress is then decomposed into volumetric and deviatoric terms in Equation 16; the shear stress varies linearly with the amount of torsion. This can then be reduced to the expression seen in Equation 17.

$$
2\left(C_{1}+C_{2}\right)=G
$$


For neo-Hookean materials, the constant $C_{2}$ is zero, simplifying the expression to yield Equation 18, which directly relates the shear modulus to the constant $C_{l}$.

$$
C_{1}=\frac{G}{2}
$$

Under physiological conditions, an isotropic material approximation seems to be appropriate [35]. However, it must be noted that biological tissues can exhibit anisotropic behaviors. Orthotropic material models can be appropriate due to alignment of fibers in biological tissues that optimize normal physiological function. Using such a model could increase accuracy. Unfortunately, one potential disadvantage of using an orthotropic material model is that it may complicate symmetry conditions; this could require a greater portion of geometry to be modeled than a simple isotropic approach, which in turn could drive up computational costs. Such models also require a greater number of physical constants to be measured and estimated. Experiments have been conducted to measure the anisotropic properties of arteries to develop these more complicated material models [35].

For increased detail, it is possible to separate the artery or plaque into distinct layers or regions. Layer specific models of the artery have been developed [49]. These require data for each layer, which may have their own unique directional material properties. This requires careful dissection and analysis of tissues. In some programs, computationally 
expensive contact algorithms may be required if several separate meshes are used, though issue can be avoided if the problem is properly defined. Some variability is present in the mechanical properties of arteries reported in literature. The shear modulus of an artery is reported to be around $150 \mathrm{kPa}$ in various models $[50,51]$. One study in a porcine model fond the stiffness to be much greater at around $230 \mathrm{kPa}$ [52], while another study found that it varied between 80 and $130 \mathrm{kPa}$ in a healthy human subject [53]. Blood vessels are given a density of about $930 \mathrm{~kg} / \mathrm{m}^{3}$ in some models $[54,55]$.

The physical properties of an atheroma can vary considerably depending on its composition, which varies from case to case as one would expect. The shear modulus has been report to be between 7 and $100 \mathrm{kPa}$ when regarding harvested plaques as being composed of isotropic neo-Hookean materials [56], and the Young's modulus has been estimated between 5 and $50 \mathrm{kPa}$ approximating the material as being linearly elastic [57]. Plaques have been modeled as incompressible materials [58, 59]. Mass density has been reported ranging from 1220 to $1450 \mathrm{~kg} / \mathrm{m}^{3}$ [60].

Blood is a fairly well characterized material when treated as a homogenous fluid. Although it is a non-Newtonian fluid, at high shear rates, it can be approximated as a Newtonian fluid that has a linear relationship between the stress and deformation as demonstrated in Equation 19, where $\mu$ is a constant when considering Newtonian fluids [61]: 


$$
\tau=\mu \frac{d u}{d y}
$$

In the larger arteries, blood viewed under various imaging techniques exhibits roughly Newtonian fluid behavior [62]. Whole blood has a viscosity on the order of about $3.8 \mathrm{mPa}-\mathrm{s}$ [63]. The density of blood is commonly given as $1060 \mathrm{~kg} / \mathrm{m}^{3}$, which is slightly greater than water [54]. Under normal physiological conditions, blood tends to exhibit laminar flow, though certain pathological conditions can cause turbulent flow to develop [64]. Laminar flow, which occurs when the ratio of inertial forces to viscous forces is very low, can be more easily predicted mathematically. Blood is often modeled as an incompressible fluid [65]. 


\section{The Role of Shear Stress}

Hemodynamics have a significant impact on the endothelial lining of mammalian arteries [62]. The endothelium provides an anticoagulant barrier that also enables physiological regulation of vasodilatation, vasoconstriction, and vascular permeability. Unsurprisingly, the endothelium also mediates the responses to cardiovascular disorders. The shear force generated by flowing blood deforms the cells, triggering chemical pathways. In healthy arteries, high shear stress can promote beneficial structural changes; however, the response changes in the early stages of cardiovascular diseases, especially when hypertension and hypercholesterolemia are present.

Shear stress is important in determining where most vascular symptoms originate; it contributes to the phenotypic changes that increase susceptibility to atherosclerosis and advance its progression. Local geometry influences the distribution and magnitude of shear stress. This can be dramatically altered by the presence of a stenotic plaque or an implanted device such as a stent. For example, considering the flow described by the Hagen-Poiseuille equation (Equation 1), the shear stress at the vessel walls can increase dramatically as the value of the radius is decreased (Equation 20).

$$
\tau=\frac{4 \mu Q}{\pi r^{3}}
$$


For healthy left coronary arteries, the magnitude of wall shear stress (WSS) is quite low. One computational study of the hemodynamics predicted WSS rarely exceeding about $4.5 \mathrm{~Pa}$ in the proximal regions of a healthy curved LAD [66]. Another study gave the normal, healthy range of WSS in the LAD as between about 1 and $4 \mathrm{~Pa}$ [67]. Greater shear forces are believed to be capable of inducing endothelial damage. It is believed that a WSS of about $35 \mathrm{~Pa}$ is sufficient to inflict such damage; this magnitude of WSS has been predicted in one model a half closed LAD [68]. 


\section{Modeling Methods and Goals}

Several models were created using COMSOL Multiphysics ${ }^{\circledR}$, a commercially available finite element analysis (FEA) and multiphysics simulation software package capable of modeling fluid flow, structural mechanics, and fluid-structure interactions. These models are of straight segments of arteries that include vessel compliance and taper. Models were created for a perfectly healthy artery segment as well as for varying degrees of stenosis. Several modeling techniques were explored.

Finite element analysis works by reducing a large problem into many, smaller and easier to solve problems. This technique uses many types of elements with different interpolation and numerical integration methods. In FEA, the governing equations are often incorporated in the calculations using their weak form. Numerical integration is then performed at the element level to establish relationships between nodes and elements. For example, in a linear solid mechanics problem, stiffness between each degree of freedom is found on the element level and compiled to the global level. The complete system of linear equations is then solved after being subjected to applied loads and boundary conditions. Increasing the number of elements in a mesh can give more reliable results, but this can come at the cost of significantly increased computational time. Non-linear problems perform this analysis iteratively until a solution satisfying a convergence criterion is found. Non-linearity can be introduced with material models, geometries, loads, and from other 
sources. Owing to the iterative nature of the solution process, this results in analysis taking longer than direct, linear matrix solutions.

COMSOL® is capable of using Arbitrary Lagrangian Eulerian (ALE) methods that contribute to its characteristic multiphysics simulation ability. The choice of coordinate systems is important for solving for the movement of matter. A Lagrangian approach, in which material points of a body are followed, is typically used when describing deformations of solid bodies. In the context of FEA, this approach tends to be computationally efficient and resolves material boundaries well [69]. However, it must be noted that the range of distortion must be limited to produce reliable results. As distortion of elements increases, their performance can dramatically decrease, leading to substantial inaccuracies. On the other hand, Eulerian methods, in which the movement of matter is tracked through fixed spatial coordinates, are often applied to fluid dynamics problems. Eulerian FEA methods overcome the deformation issue but are more computationally complex [69]. ALE methods use both coordinate systems to move in an arbitrary manner, often using a Lagrangian method in the structure and Eulerian method in the fluid with a transition between the two domains to account for fluidstructure interactions [69]. Employing ALE methods introduces nonlinearity, which requires an iterative solution method.

Model geometry was created as three-dimensional parts using Dassault Systèmes Solidworks ${ }^{\circledR}$. Quarter symmetry was used to reduce 
the adequate mesh sizes required to produce consistent results, allowing for more efficient computational times. A simple initial model not featuring taper was created with a radius of $1.5 \mathrm{~mm}$ to validate the accuracy of predictions given by COMSOL $₫$ in comparison to analytical solutions for fluid dynamic and solid mechanical quantities of interest. After verifying these predictions, material properties were selected for the solid mechanics domain to produce the proper amount of distension. A Young's modulus of $50 \mathrm{kPa}$ was assigned to all lesions; due to time constraints, other values were not tested.

Next, a segment of the LAD was modeled as a straight geometry with a proximal radius of $1.75 \mathrm{~mm}$ (corresponding to the larger diameters reported for the LAD) and a distal radius of $1.25 \mathrm{~mm}$ to create a taper. As lesions can vary considerably based on the individual case, an arbitrary length of $9 \mathrm{~mm}$ and shape was selected for the stenosis in the diseased models. The arteries were modeled homogenously with a single layer. Plaques for the diseased models were given their own unique geometry. These plaques were created to that they produce a $25 \%, 50 \%$, and $75 \%$ reduction in the lumen radius; again, as lesions can vary significantly between cases, these sizes were arbitrarily selected. Lumens were also separately modeled to accommodate the appropriate geometries. These unmeshed geometries are shown in Figures 7 through 9. 


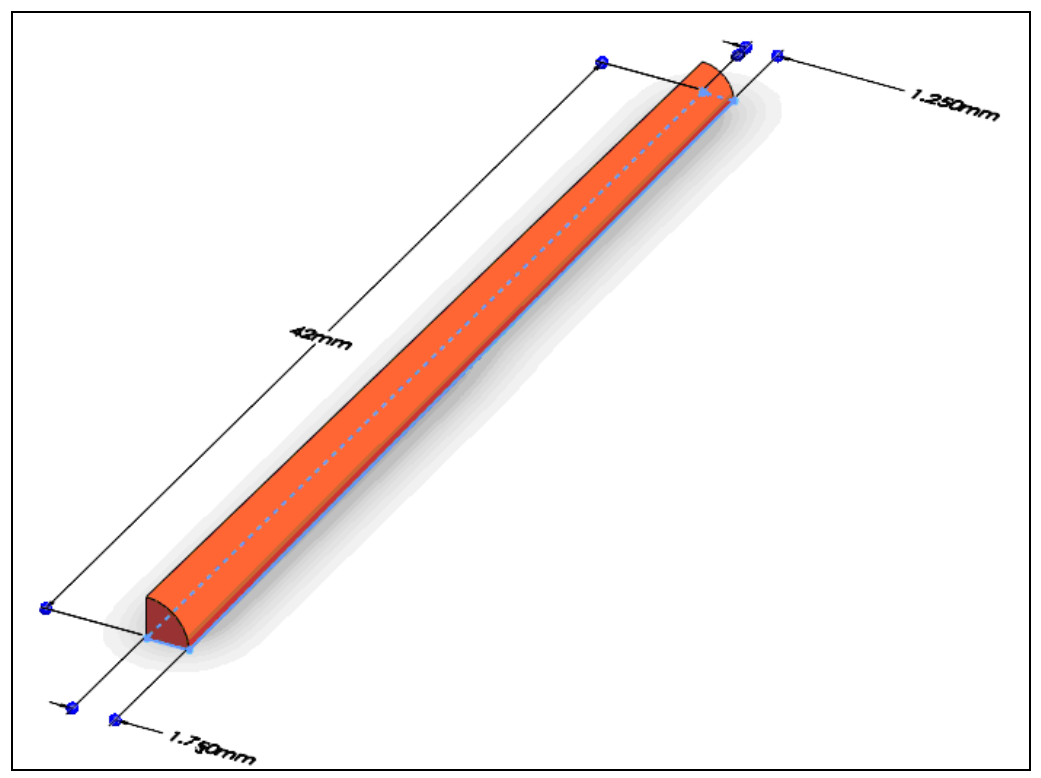

Figure 7: Trimetric projection of the tapered geometry used to create the lumen for the healthy artery model.

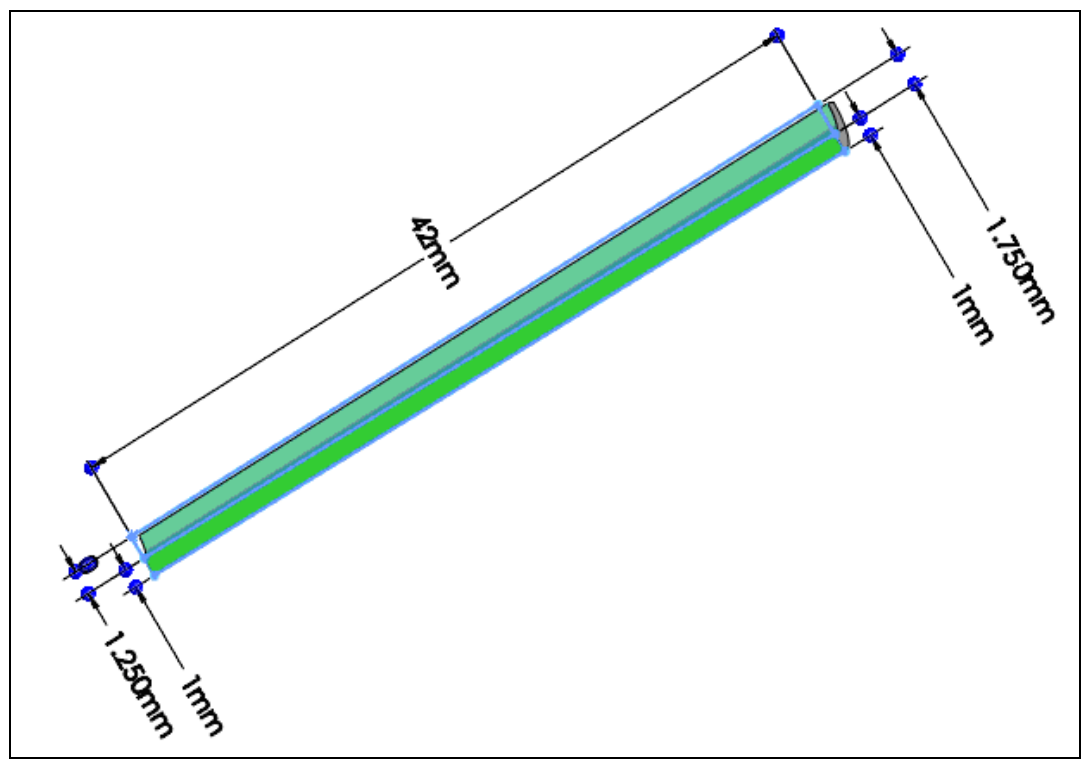

Figure 8: Trimetric projection of the tapered geometry used to create the artery wall for the healthy artery model. 


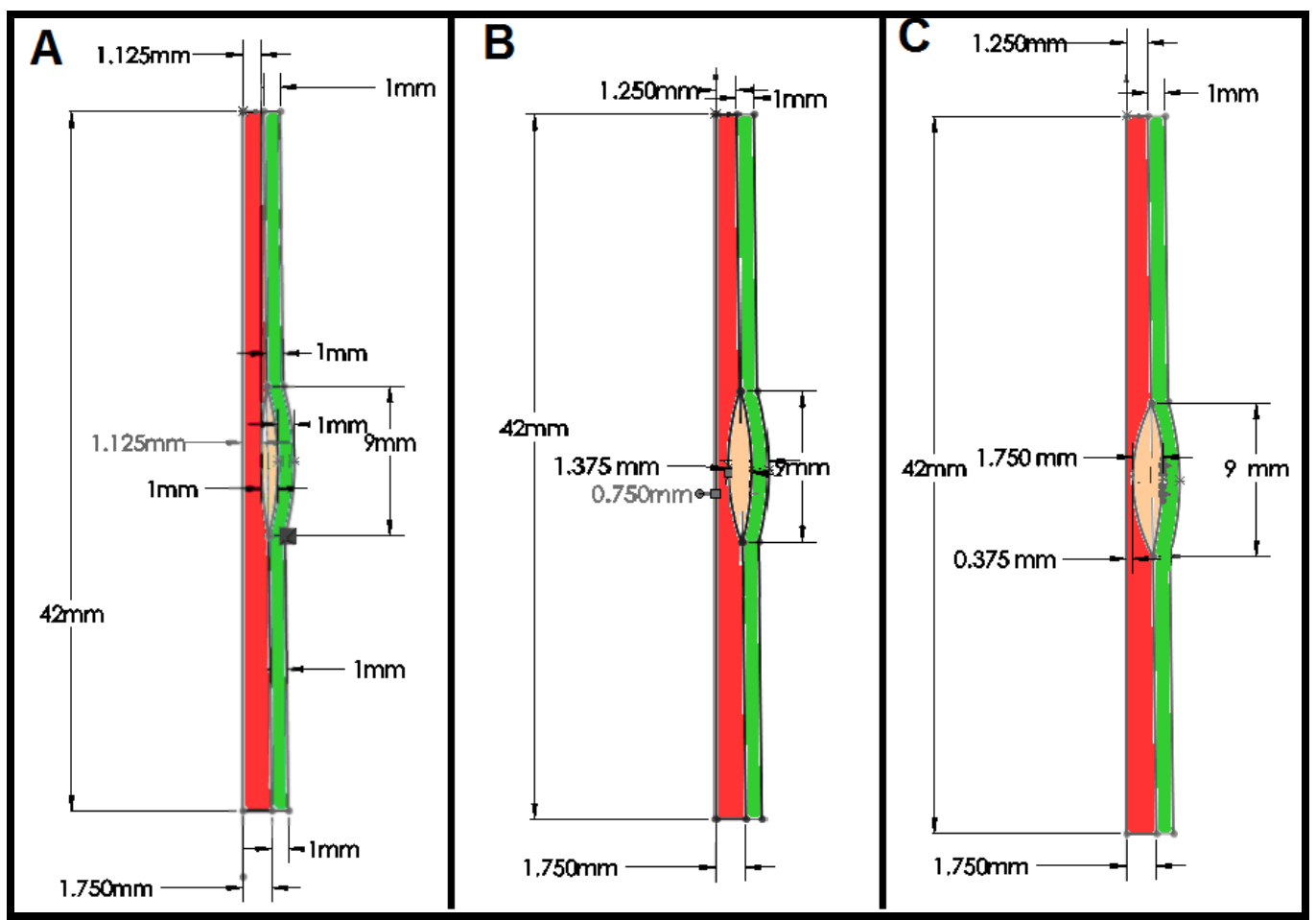

Figure 9: Two-dimension view of geometry revolved to create diseased artery model for (a) a $25 \%$, (b) $50 \%$, and (c) $75 \%$ reduction of lumen radius. (Red-Lumen, Yellow-Plaque, GreenArtery Wall)

Geometries were then imported and used to create meshes in COMSOL $®$. COMSOL $®$ has integrated tools for refining meshes, though they are somewhat rudimentary. With these artery geometries, the default resolution remained low in the radial direction; by adjusting the mesh size parameters available by default, an increase in the radial resolution could come at the cost of needlessly increasing the resolution in the axial direction. Fortunately, COMSOL® is able to import meshes from other more specialized FEA preprocessors that are compatible with the NASTRAN, VRML, and STL mesh file formats. An alternative method to boost radial resolution was found; users may specify a greater number of elements on the surfaces of individual geometries through the addition of "boundary layers". With the initial testing complete, a convergence 
study was conducted to ensure the models produced consistent results and to refine meshes as needed.

The loading of the vessel was set by defining the pressures at the proximal and distal ends of the lumen segment. The pressure waveform used in time dependent studies was derived from a three element Windkessel model as shown in Figure 5. To determine the difference in pressure between the inlet and outlet of the artery, the Hagen-Poiseuille (Equation 1) was first used, approximating the artery as a cylinder of radius $1.5 \mathrm{~mm}$ with an average velocity of $20.5 \mathrm{~cm} / \mathrm{s}$; this corresponds to a pressure drop of approximately $116 \mathrm{~Pa}$. As this does not account for the taper or increased resistance of stenosed arteries, this pressure drop was later adjusted through trial and error to produce the average amount of flow for each model as described in Table 1 unless explicitly stated otherwise. In the studies on coronary flow reserve (CFR) in coronary arteries previously described in Chapter VI, it was suggested that there is little decrease in flow for arteries that are less than $70 \%$ occluded; more severely stenosed arties had their maximum flow fall profoundly compared to the healthier counterparts. The flow rates shown in Table 1 were selected somewhat arbitrarily to show a minor decrease in flow for the three models with less than $70 \%$ stenosis; the model featuring $75 \%$ reduction of lumen radius was given a flow rate based upon the decreased CFR reported in the previously mentioned studies. As the coronary circulation is fed from the aorta shortly as it leaves the heart, the total 
pressure magnitudes experienced by the artery wall were assumed to be approximately those seen in the larger systemic arteries.

Table 1: Average flow rate assigned to each model and associated pressure drop across the artery.

\begin{tabular}{|c|c|c|c|}
\hline $\begin{array}{c}\text { Lumen Radius } \\
\text { Reduction }\end{array}$ & \multicolumn{2}{|c|}{ Average Flow Rate } & $\begin{array}{c}\text { Pressure } \\
\text { Drop }\end{array}$ \\
\hline$(\%)$ & $\mathrm{mL} / \mathrm{s}$ & $(\%)$ & $\mathrm{Pa}$ \\
\hline $0 \%$ & 1.44906 & $100 \%$ & 135 \\
\hline $25 \%$ & 1.304154 & $90 \%$ & 150 \\
\hline $50 \%$ & 1.231701 & $85 \%$ & 260 \\
\hline $75 \%$ & 0.869436 & $60 \%$ & 1200 \\
\hline
\end{tabular}

Relatively simple boundary conditions were implemented in these models. For the solid mechanics domain, the distal end of the artery was axially fixed. The proximal end was also axially fixed, but included a prescribed displacement to simulate the in-situ stretch seen in arteries; a 5\% stretch was assigned to all models. Due to Poisson's effects and the conservation of volume, this results in inward forces to close the vessel and help oppose the outward push caused by the blood.

For the fluid domains, two physics modules were used and compared in a series of tests. The "Laminar Flow" fluid physics module is somewhat more accurate and uses the relationships shown in Equation 21 and Equation 22 to solve the dependent variables of the fluid system:

$$
\begin{gathered}
\rho(\bar{u} \cdot \bar{\nabla} \bar{u})=\bar{\nabla} \cdot\left[-p \overline{\bar{I}}+\mu\left(\bar{\nabla} \bar{u}+(\bar{\nabla} \bar{u})^{T}\right)-\frac{2}{3} \mu(\bar{u} \cdot \bar{\nabla}) \overline{\bar{I}}\right]+\bar{F} \\
\frac{\partial \rho}{\partial t}+\bar{\nabla} \cdot(\rho \bar{u})=0
\end{gathered}
$$

Here, $\rho$ is the material mass density, $u$ is the fluid velocity field, $p$ is the pressure, $\mu$ is the viscosity, $F$ is a volume force, and $I$ is the identity tensor. 
In contrast, there is the "Creep Flow" module that uses simplified relationships shown in Equation 23 and Equation 24. Given its simpler nature, solving for dependent variables is somewhat quicker in comparison, though likely less accurate and reliable. Results derived with each technique were compared.

$$
\begin{gathered}
0=\bar{\nabla} \cdot\left[-p \overline{\bar{I}}+\mu\left(\bar{\nabla} \bar{u}+(\bar{\nabla} \bar{u})^{T}\right)-\frac{2}{3} \mu(\bar{u} \cdot \bar{\nabla}) \overline{\bar{I}}\right]+\bar{F} \\
\bar{\nabla} \cdot(\bar{u})=0
\end{gathered}
$$

After comparing the two different approaches to modeling the flow of blood, a comparison of the distension and radial stress distribution through the arterial wall was performed for each model using a linear elastic and hyperelastic approach. Material properties were selected in the previously described initial testing. The fluid domain was modeled with the "Creep Flow" module to speed up calculation. A load corresponding to mean arterial pressure (MAP) was used along with a pressure drop across the artery producing the average flow.

Finally, a simulation of the arteries undergoing a single cardiac cycle was created. The pressure waveform used was that seen in the 3element Windkessel model pictured in Figure 5. The pressure drop was varied by using a piecewise defined sinusoid that produced average flow values seen in Table 1 and with amplitudes consistent with the coronary flow reserve ratios from the previously discussed studies.

Although there are limitations in these models, there are still ways to validate their performance. In patients with coronary artery disease, 
one should expect to see a difference in vessel lumen area between systole and diastole on the order of about $10 \%$ [70,71], with possible variation based on the nature of any plaques. This rough estimation is not much different than which has been observed in left anterior descending arteries that appear normal under angiogram [72]. In the case of shear stress, very low magnitudes-no greater than about $5 \mathrm{~Pa}-$ are expected to appear for the healthy artery model. Shear stress should rise at the sites of narrowing, and should grow profoundly as the lumen radius decreases. 


\section{Results and Discussion}

\section{A. Initial Testing}

Before thoroughly examining the tapered artery models, a few of quantities were predicted analytically and compared to the result given in COMSOL $®$ for a cylindrical vessel with a $1.5 \mathrm{~mm}$ radius with a mesh of approximately 20,000 elements total. Results are summarized in Table 2. First, a pressure difference between the inlet and outlet was set as $116 \mathrm{~Pa}$; the resultant average fluid velocity was compared to that predicted by the Hagen-Poiseuille equation (Equation 1). The error was slightly greater than one percent. Next, wall shear stress for these conditions was calculated using Equation 19; agreement between the two predictions was excellent.

Table 2: Comparison of analytical and numerical predictions of several different quantities in the simplified, non-tapered model of a healthy coronary artery.

\begin{tabular}{|c|c|c|c|}
\hline Quantity & $\begin{array}{c}\text { Analytical } \\
\text { Prediction }\end{array}$ & $\begin{array}{c}\text { COMSOL® } \\
\text { Prediction }\end{array}$ & $\begin{array}{c}\text { Error in COMSOL® } \\
\text { Prediction }\end{array}$ \\
\hline Average Velocity & $0.20266 \mathrm{~m} / \mathrm{s}$ & $0.20023 \mathrm{~m} / \mathrm{s}$ & $-1.20 \%$ \\
\hline Artery Wall Shear Stress & $2.05357 \mathrm{~Pa}$ & $2.04884 \mathrm{~Pa}$ & $-0.23 \%$ \\
\hline Hoop Stress at Outer Radius & $11250 \mathrm{~Pa}$ & $11086 \mathrm{~Pa}$ & $-1.46 \%$ \\
\hline
\end{tabular}

To verify the performance of the solid mechanics module, the pressure on the inner wall was set to $10 \mathrm{kPa}$ while the outer wall was left unloaded. No axial stretch was applied. Hoop stress was predicted at the outer radius of the artery wall using the thick walled cylinder stress distribution (Equation 25).

$$
\sigma_{\theta}=\frac{p_{i} R_{i}^{2}-p_{o} R_{o}^{2}}{R_{o}^{2}-R_{i}^{2}}-\frac{\left(p_{o}-p_{i}\right) R_{i}^{2} R_{o}^{2}}{r\left(R_{o}^{2}-R_{i}^{2}\right)}
$$


In this equation, $p_{i}$ is the internal pressure, $p_{o}$ is the external pressure, $R_{i}$ is the internal radius, $R_{o}$ is the external radius, and $r$ is the radial position the hoop stress is being calculated at. Agreement was excellent at this point, though a more refined mesh may be needed for a smoother distribution of hoop stress throughout the entire radius.

This simple model was also used to explore the values of material properties of the artery walls that produce the most realistic distention. A $5 \%$ axial stretch was applied, which is near the value seen in human specimens mentioned previously discussed studies. A constant pressure was applied to simulate diastole for each value of the shear modulus; the process was repeated with a pressure corresponding to systole. The animal studies previously mentioned in this paper gave values of the shear modulus around $140 \mathrm{kPa}$. For the linear elastic model, which has results summarized in Table 3, this would appear to be stiffer than expected; a value of about $100 \mathrm{kPa}$ instead produces the roughly $10 \%$ change in lumen area that studies have found for this coronary artery.

Table 3: Distension and area change between systole and diastole in the non-tapered healthy artery model using an isotropic linear elastic material with varied shear modulus.

\begin{tabular}{|c|c|c|c|c|c|c|c|}
\hline \multirow{2}{*}{$\begin{array}{l}\text { Shear } \\
\text { Modulus } \\
\text { (kPa) }\end{array}$} & \multicolumn{2}{|c|}{$\begin{array}{l}\text { Displacement (m) } \\
\text { for a load of }\end{array}$} & \multicolumn{2}{|c|}{$\begin{array}{l}\text { Lumen Radius } \\
\text { (mm) for load of: }\end{array}$} & \multicolumn{2}{|c|}{$\begin{array}{c}\text { Lumen Area }\left(\mathrm{mm}^{2}\right) \\
\text { for a load of: }\end{array}$} & \multirow{2}{*}{$\begin{array}{c}\text { Lumen Area } \\
\text { Change } \\
(\%)\end{array}$} \\
\hline & $10 \mathrm{kPa}$ & $16 \mathrm{kPa}$ & $10 \mathrm{kPa}$ & $16 \mathrm{kPa}$ & $10 \mathrm{kPa}$ & $16 \mathrm{kPa}$ & \\
\hline 75 & 1.22E-04 & 2.17E-04 & 1.62 & 1.72 & 8.26 & 9.26 & $12.05 \%$ \\
\hline 100 & 8.28E-05 & 1.54E-04 & 1.58 & 1.65 & 7.87 & 8.60 & $9.23 \%$ \\
\hline 125 & 5.95E-05 & 1.17E-04 & 1.56 & 1.62 & 7.64 & 8.21 & $7.48 \%$ \\
\hline 150 & 4.41E-05 & $9.20 \mathrm{E}-05$ & 1.54 & 1.59 & 7.49 & 7.96 & $6.29 \%$ \\
\hline 175 & 3.32E-05 & 7.43E-05 & 1.53 & 1.57 & 7.39 & 7.79 & $5.44 \%$ \\
\hline 200 & 2.51E-05 & 6.12E-05 & 1.53 & 1.56 & 7.31 & 7.66 & $4.79 \%$ \\
\hline
\end{tabular}


In contrast, for the neo-Hookean hyperelastic model, a shear modulus of about $125 \mathrm{kPa}$ resulted in the roughly a $10 \%$ change in lumen area between the high and low pressures; this is not too far from the values obtained from most of the studies conducted to characterize this parameter. The resultant changes in area for each value are summarized in Table 4.

\begin{tabular}{|c|c|c|c|c|c|c|c|}
\hline \multirow{2}{*}{$\begin{array}{c}\text { Shear } \\
\text { Modulus } \\
\text { (kPa) }\end{array}$} & \multicolumn{2}{|c|}{$\begin{array}{l}\text { Displacement (m) } \\
\text { for a load of }\end{array}$} & \multicolumn{2}{|c|}{$\begin{array}{l}\text { Lumen Radius } \\
\text { (mm) for load of: }\end{array}$} & \multicolumn{2}{|c|}{$\begin{array}{l}\text { Lumen Area }\left(\mathrm{mm}^{2}\right) \\
\text { for a load of: }\end{array}$} & \multirow{2}{*}{$\begin{array}{c}\text { Lumen Area } \\
\text { Change } \\
(\%)\end{array}$} \\
\hline & $10 \mathrm{kPa}$ & $16 \mathrm{kPa}$ & $10 \mathrm{kPa}$ & $16 \mathrm{kPa}$ & $10 \mathrm{kPa}$ & $16 \mathrm{kPa}$ & \\
\hline 100 & $1.05 E-04$ & 2.13E-04 & 1.61 & 1.71 & 8.09 & 9.21 & $13.83 \%$ \\
\hline 125 & 7.44E-05 & 1.53E-04 & 1.57 & 1.65 & 7.79 & 8.59 & $10.28 \%$ \\
\hline 150 & 5.49E-05 & 1.17E-04 & 1.55 & 1.62 & 7.60 & 8.22 & $8.20 \%$ \\
\hline 175 & 4.16E-05 & 9.33E-05 & 1.54 & 1.59 & 7.47 & 7.98 & $6.82 \%$ \\
\hline 200 & 3.19E-05 & 7.60E-05 & 1.53 & 1.58 & 7.37 & 7.80 & $5.84 \%$ \\
\hline
\end{tabular}

\section{B. Convergence Study}

A simple convergence study was conducted for each tapered artery model created. A constant loading condition was used in each case.

Several quantities in both the fluid and solid domains were evaluated at points throughout the models using the same loading conditions and material properties.

First, convergence was examined in the models using linear elastic materials and the "Creep Flow" fluid physics module. These results can be seen in Table 5. The healthy model was adjusted first. While the solid mechanics quantities converged quickly, there was still some variability in one calculated wall shear stress quantity. Although increasing the total number of elements was effective, it was subsequently found that refining 
the meshes by using additional boundary layers in the fluid domain near the artery wall could allow for consistent results with fewer than 20,000 elements.

Table 5: Convergence of several quantities in each tapered artery model using linear elasticity and creep fluid flow.

\begin{tabular}{|c|c|c|c|c|c|c|c|}
\hline Model & $\begin{array}{l}\text { Total } \\
\text { Number of } \\
\text { Elements }\end{array}$ & $\begin{array}{c}\text { Distal Point } \\
\text { X- } \\
\text { displacement } \\
\text { (m) }\end{array}$ & $\begin{array}{c}\text { Middle Point } \\
\begin{array}{c}y- \\
\text { displacement }\end{array} \\
\text { (m) }\end{array}$ & $\begin{array}{c}\text { Proximal Point } \\
x- \\
\text { displacement } \\
\text { (m) }\end{array}$ & $\begin{array}{c}\text { WSS at } \\
\text { Point } 1 \\
\text { (Pa) }\end{array}$ & $\begin{array}{c}\text { WSS at } \\
\text { Point } 2 \\
\text { (Pa) }\end{array}$ & $\begin{array}{c}\text { Velocity at } \\
\text { Center of } \\
\text { Lumen } \\
(\mathrm{m} / \mathrm{s})\end{array}$ \\
\hline \multirow{3}{*}{ Healthy } & 20,218 & 5.87E-05 & 1.02E-04 & 8.25E-05 & 1.98191 & 4.54414 & -0.51162 \\
\hline & 41,549 & 5.88E-05 & 1.02E-04 & 8.25E-05 & 1.98252 & 5.17117 & -0.50783 \\
\hline & 74,566 & 5.89E-05 & 1.02E-04 & 8.23E-05 & 1.98268 & 5.60927 & -0.50663 \\
\hline \multirow{2}{*}{$\begin{array}{c}25 \% \\
\text { Radius } \\
\text { Reduction }\end{array}$} & 17,837 & 1.42E-04 & 1.04E-04 & 4.01E-05 & 3.50624 & 2.31081 & -0.23983 \\
\hline & 54,914 & 1.43E-04 & 1.04E-04 & 4.03E-05 & 3.50658 & 2.31231 & -0.24129 \\
\hline \multirow{2}{*}{$\begin{array}{c}50 \% \\
\text { Radius } \\
\text { Reduction }\end{array}$} & 16,657 & 7.68E-05 & 1.04E-04 & 4.02E-05 & 5.83328 & 1.23513 & -0.12806 \\
\hline & 39,949 & 7.70E-05 & 1.03E-04 & 4.01E-05 & 5.82799 & 1.23557 & -0.12891 \\
\hline \multirow{2}{*}{$\begin{array}{c}75 \% \\
\text { Radius } \\
\text { Reduction }\end{array}$} & 17,897 & $3.28 \mathrm{E}-06$ & 1.04E-04 & 4.02E-05 & 7.49995 & 0.18601 & -0.01934 \\
\hline & 38,917 & 3.19E-06 & 1.04E-04 & 4.02E-05 & 7.60223 & 0.18617 & -0.01946 \\
\hline
\end{tabular}

Next, convergence was examined when using the neo-Hookean

material models. The fluid domains still employed the "Creep Flow"

physics used when examining the convergence of the linear elastic

models. Consistent results could be found in the solid mechanics domain

with about 7,000 elements in that domain. These results are summarized

in Table 6.

Table 6: Convergence of several displacement quantities in each tapered artery model using neo-Hookean materials and creep fluid flow.

\begin{tabular}{|c|c|c|c|c|}
\hline Model & $\begin{array}{c}\text { Total Number of } \\
\text { Elements In Solid } \\
\text { Mechanics Domain }\end{array}$ & $\begin{array}{c}\text { Proximal Point } \\
\text { x-displacement }\end{array}$ & $\begin{array}{c}\text { Distal Point } \\
\text { y-displacement }\end{array}$ & $\begin{array}{c}\text { Center Point } \\
\text { x-displacement }\end{array}$ \\
\hline Healthy & 7,461 & $1.37 \mathrm{E}-04$ & $9.25 \mathrm{E}-05$ & $3.96 \mathrm{E}-05$ \\
\hline & 17,881 & $1.38 \mathrm{E}-04$ & $9.25 \mathrm{E}-05$ & $3.97 \mathrm{E}-05$ \\
\hline $\begin{array}{c}25 \% \text { Radius } \\
\text { Reduction }\end{array}$ & 6,592 & $1.54 \mathrm{E}-04$ & $9.21 \mathrm{E}-05$ & $1.29 \mathrm{E}-04$ \\
\hline $\begin{array}{c}50 \% \text { Radius } \\
\text { Reduction }\end{array}$ & 14,643 & $1.57 \mathrm{E}-04$ & $9.29 \mathrm{E}-05$ & $1.31 \mathrm{E}-04$ \\
\hline $75 \%$ Radius & 7,182 & $1.62 \mathrm{E}-04$ & $9.31 \mathrm{E}-05$ & $1.35 \mathrm{E}-04$ \\
\hline Reduction & 14,963 & $1.55 \mathrm{E}-04$ & $9.24 \mathrm{E}-05$ & $1.33 \mathrm{E}-04$ \\
\hline
\end{tabular}


After testing the hyperelastic material models, convergence was examined using the "Laminar Flow" physics module for the fluid domain. The linear elastic material model was used for the solid mechanics domain. Using the insights gained in the previous convergence tests, the meshes were refined. It was determined that consistent results could be achieved at several points when approximately 6,000 elements are in the fluid domain. These results are summarized in Table 7.

\begin{tabular}{|c|c|c|c|c|}
\hline Model & $\begin{array}{l}\text { Total Number of } \\
\text { Elements in Fluid } \\
\text { Mechanics Domain }\end{array}$ & $\begin{array}{l}\text { Distal Center } \\
\text { Velocity } \\
(\mathrm{m} / \mathrm{s})\end{array}$ & $\begin{array}{c}\text { Distal Point } \\
\text { Wall Shear Stress } \\
(\mathrm{Pa})\end{array}$ & $\begin{array}{c}\text { Proximal Point } \\
\text { Pressure } \\
(\mathrm{Pa}) \\
\end{array}$ \\
\hline \multirow{2}{*}{ Healthy } & 6,063 & -0.41955 & 1.86704 & $15,970.08$ \\
\hline & 11,327 & -0.42787 & 1.80367 & $15,969.47$ \\
\hline \multirow{2}{*}{$\begin{array}{l}25 \% \text { Radius } \\
\text { Reduction }\end{array}$} & 5,861 & -0.57158 & 1.37641 & $15,980.00$ \\
\hline & 12,121 & -0.55884 & 1.49214 & $15,980.00$ \\
\hline \multirow{2}{*}{$\begin{array}{l}50 \% \text { Radius } \\
\text { Reduction }\end{array}$} & 6,061 & -0.31788 & 0.73121 & $15,989.11$ \\
\hline & 12,916 & -0.29984 & 0.79513 & $15,989.29$ \\
\hline \multirow{2}{*}{$\begin{array}{l}75 \% \text { Radius } \\
\text { Reduction }\end{array}$} & 6,129 & -0.04481 & 0.1081 & $15,998.63$ \\
\hline & 11,964 & -0.04669 & 0.11476 & $15,998.39$ \\
\hline
\end{tabular}

Overall, consistent results appear with slightly less than fifteen thousand elements are present in the model. It was important to have a slightly greater amount of detail in the radial direction when refining the meshes. It should be noted that the mesh refinement tools integrated into COMSOL® are somewhat rudimentary, which accounts for some of the variability of the number of elements tested in each case. 


\section{Laminar Flow Versus Creep Flow Computations}

A comparison of the wall shear stresses predicted by the "Creep Flow" and "Laminar Flow" physics modules of COMSOL® was conducted for each tapered artery model. The pressure drop across the artery was selected to mimic average physiological flow as previously described; these parameters are listed on the graphs comparing the shear stress distributions (Figures 10 through 13).

The predictions generally agree and show the same behavior. In the healthy artery, the prediction for creeping flow was greater than that for laminar flow. This is also seen in the $25 \%$ reduced radius model, though the agreement between the two methods is better than the previous case. The model with the $50 \%$ radius reduction continues this trend, and has the greatest agreement between the laminar flow and creep flow

predictions. Agreement between the laminar flow and creep flow predictions was excellent in the un-occluded section of the artery with $75 \%$ reduced radius, though the shear stress at the center of the lesion was predicted to be considerably higher in the creep flow version of the model. Both physics modules showed somewhat dramatic changes in wall shear stress at the sites of transitions between the lesion and healthy portions of the artery, as well at the inlet and outlet of the artery. 


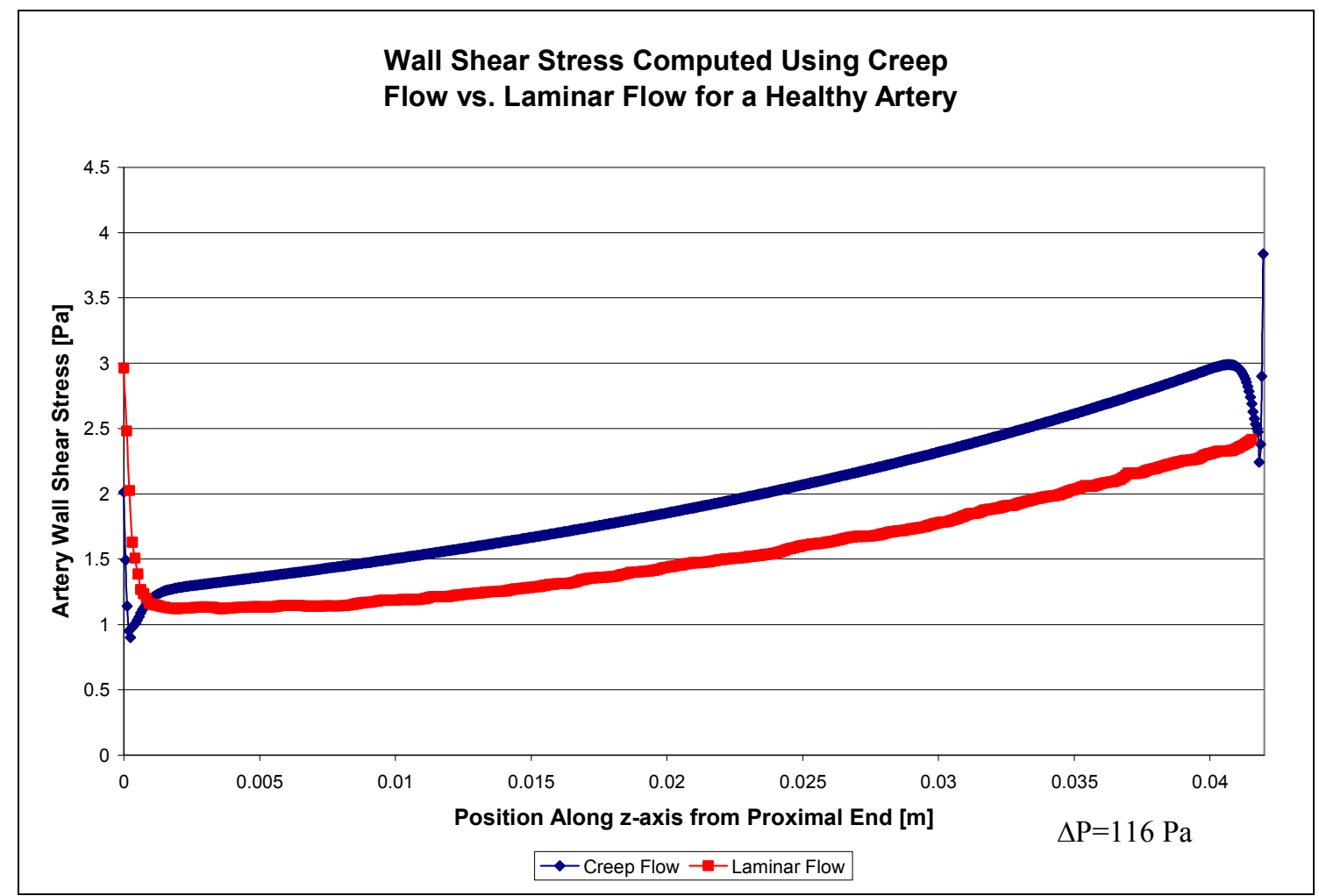

Figure 10: Comparison of predictions for wall shear stress using "Creep Flow" and "Laminar Flow" physics modules in a model of a healthy tapered coronary artery. 


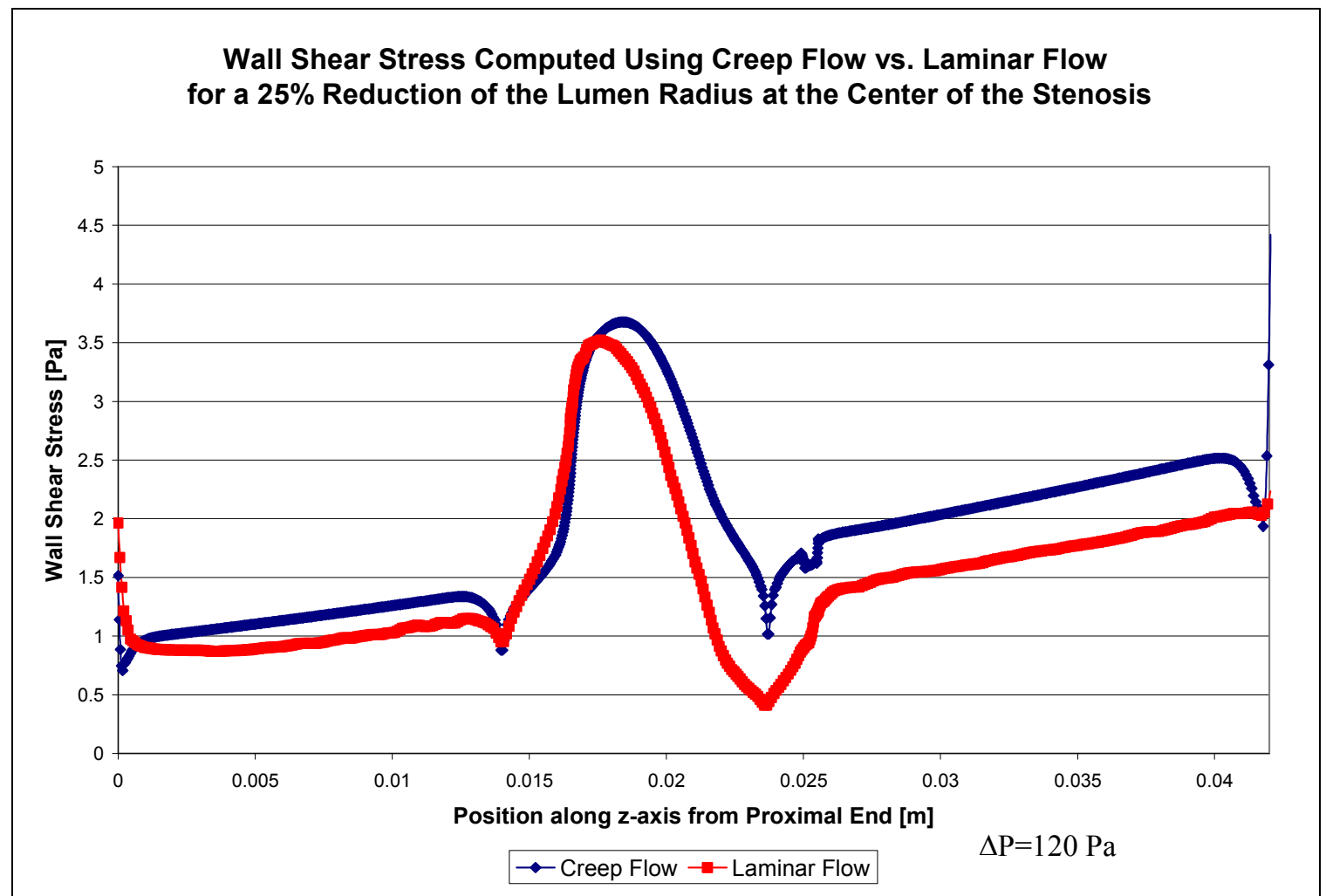

Figure 11: Comparison of predictions for wall shear stress using "Creep Flow" and "Laminar Flow" physics modules in a model of a tapered coronary artery with a $25 \%$ reduction in lumen radius. 


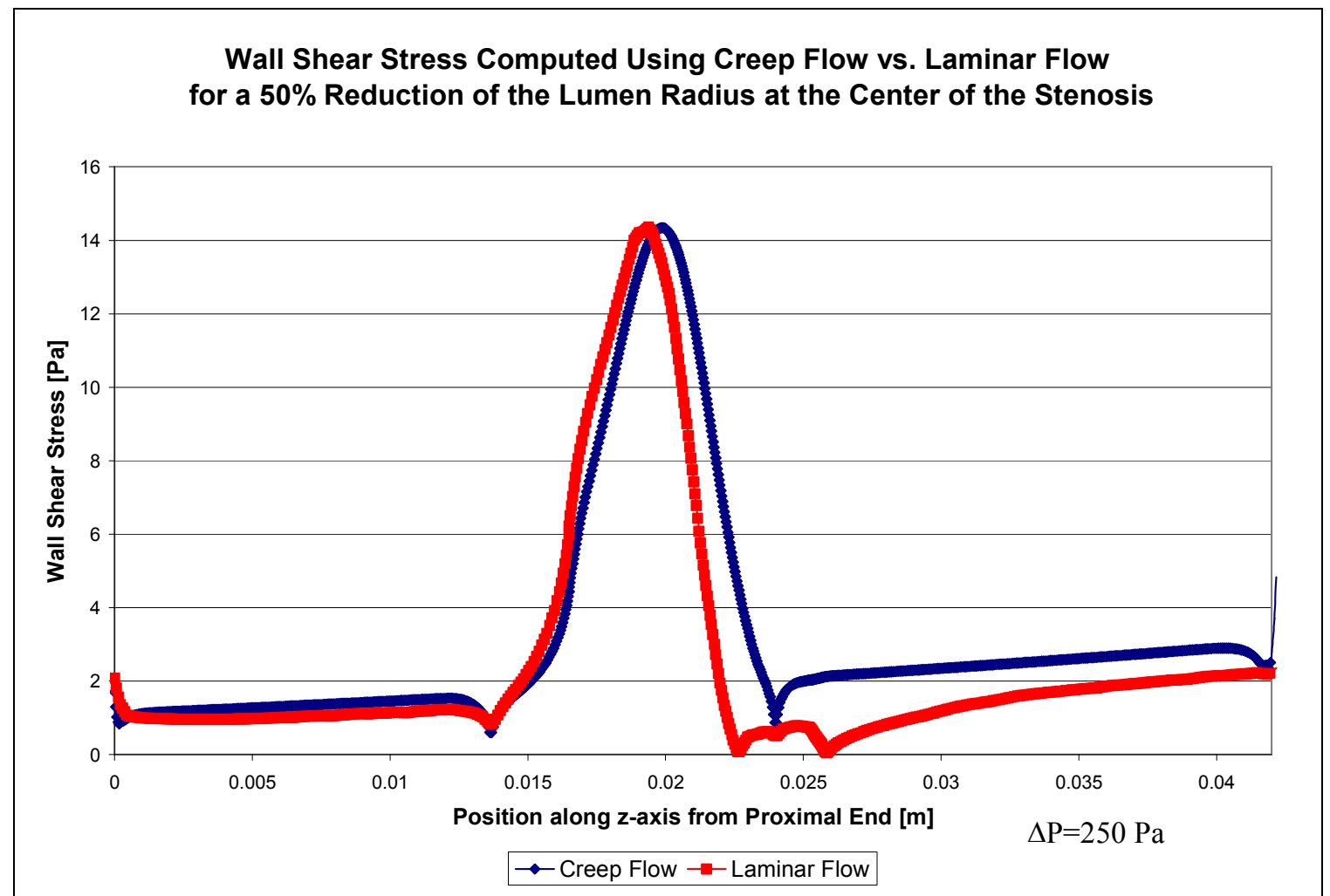

Figure 12: Comparison of predictions for wall shear stress using "Creep Flow" and "Laminar Flow" physics modules in a model of a tapered coronary artery with a $50 \%$ reduction in lumen radius. 


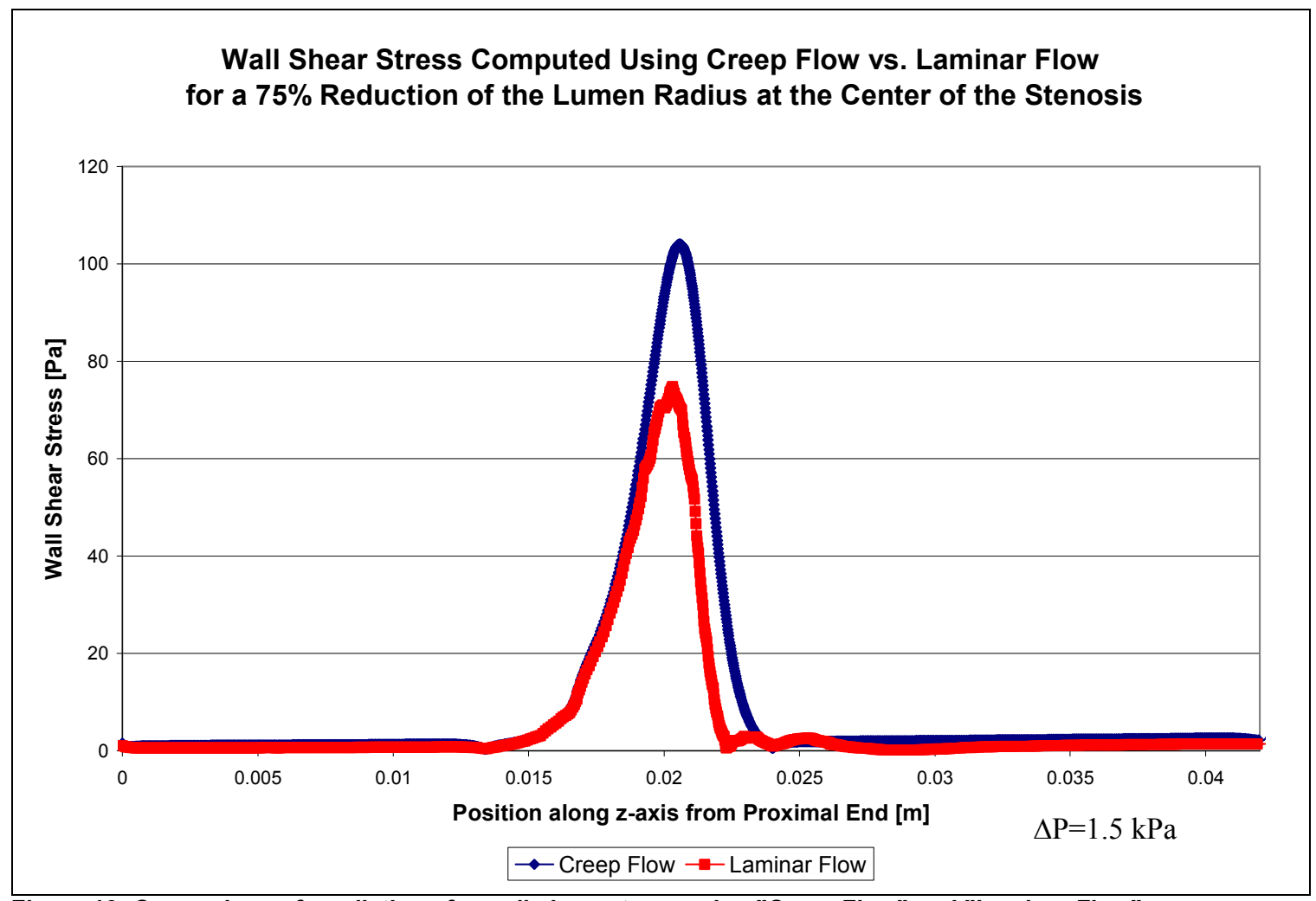

Figure 13: Comparison of predictions for wall shear stress using "Creep Flow" and "Laminar Flow" physics modules in a model of a tapered coronary artery with a $75 \%$ reduction in lumen radius.

\section{Linear Elastic Versus Neo-Hookean Material Models}

A comparison of the behaviors seen when using either neo-

Hookean or linearly elastic material models was performed. Stiffness

values for the artery wall were selected based on the distension seen in the

initial testing previously described. Figure 14 shows the change in area

between systole and diastole along the entire length of the healthy artery 
model. The overall behavior is remarkably similar in each case. The neo-

Hookean model's response was a little stiffer, but this may be attributed to the selection of material constants.

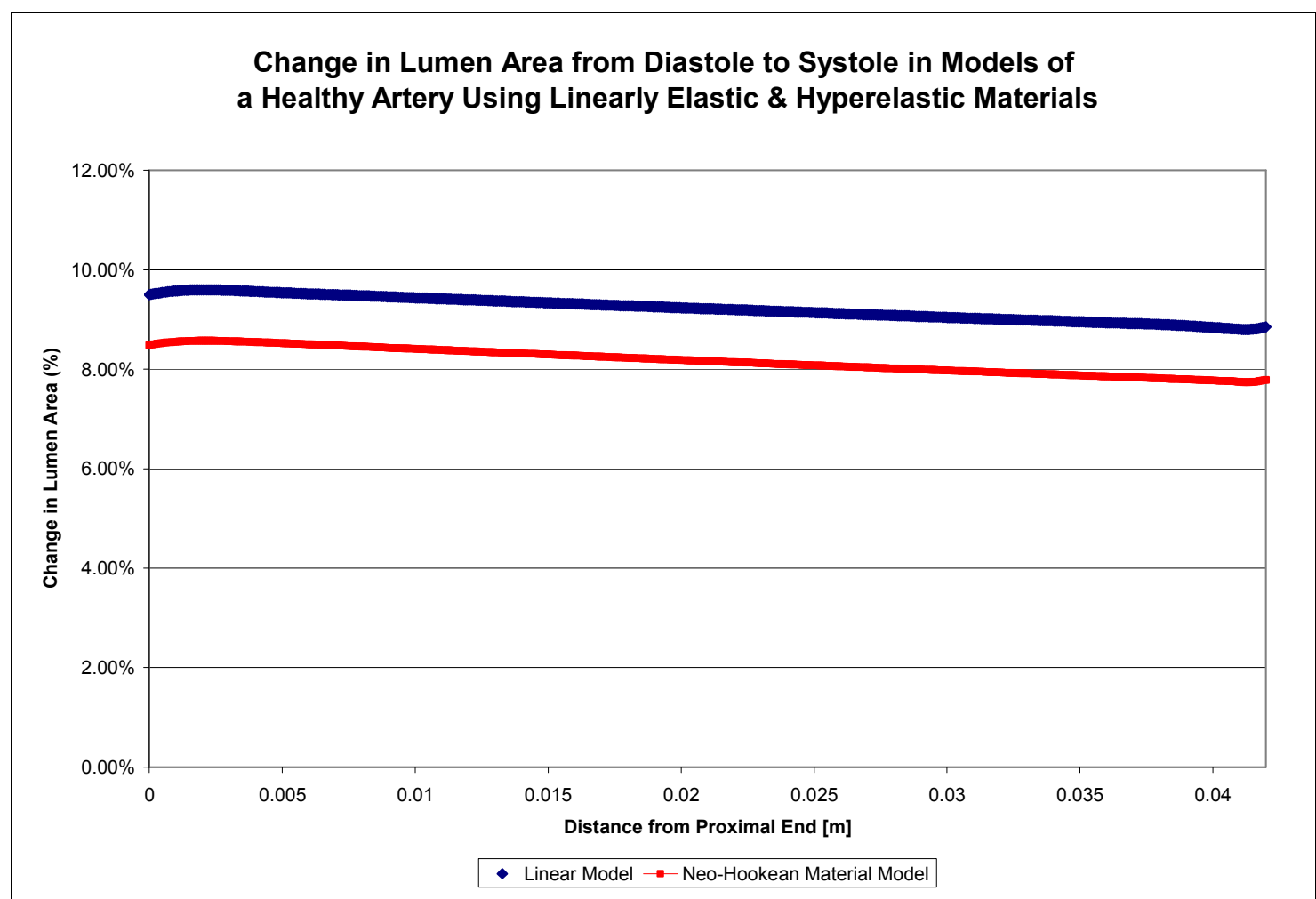

Figure 14: Comparison of lumen area change between a healthy tapered artery modeled with an isotropic linearly elastic material and an isotropic neo-Hookean material model.

Next, the distribution of radial stress through the artery wall near the center of the vessels (where the stenosis occurs in the diseased models) was compared between the two material models using each set of geometries. These results are shown in Figures 15 through 18. The results are overall similar, though the neo-Hookean approach produced much smoother curves for the distribution of radial stress than the linear isotropic models using the same meshes. The radial stresses at the boundaries of the outer artery wall and inner surface of the plaque 
corresponded to the loading conditions as would be expected. The neo-

Hookean models generally experienced slightly greater stresses. Given

the nature of biological tissues, this hyper-elastic material model would be

more appropriate, though it adds a source of non-linearity to the problem,

in turn increasing computational time as an iterative solution method

becomes necessary.

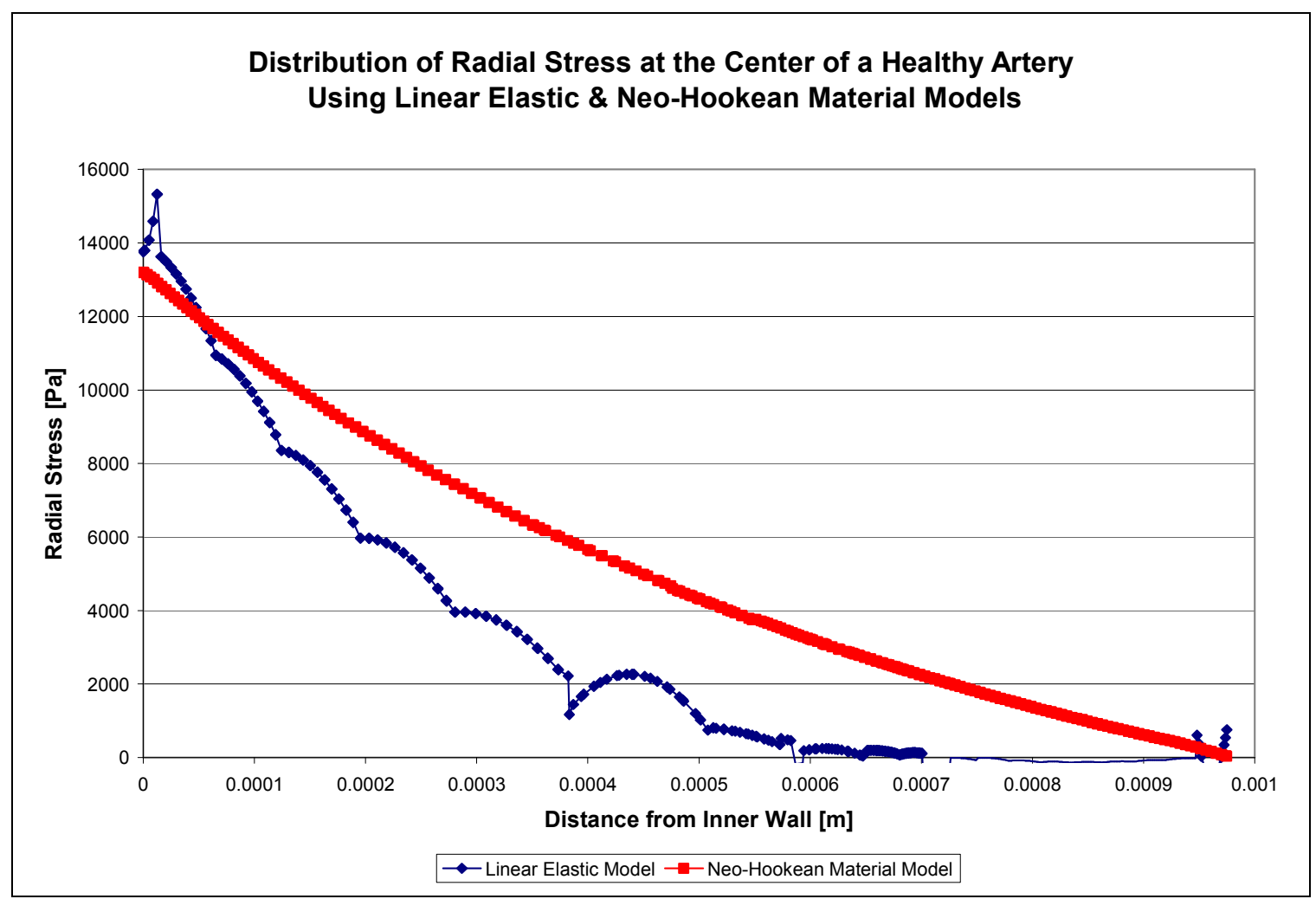

Figure 15: Comparison of radial stress distribution between a healthy tapered artery modeled with an isotropic linearly elastic material and an isotropic neo-Hookean material model. 


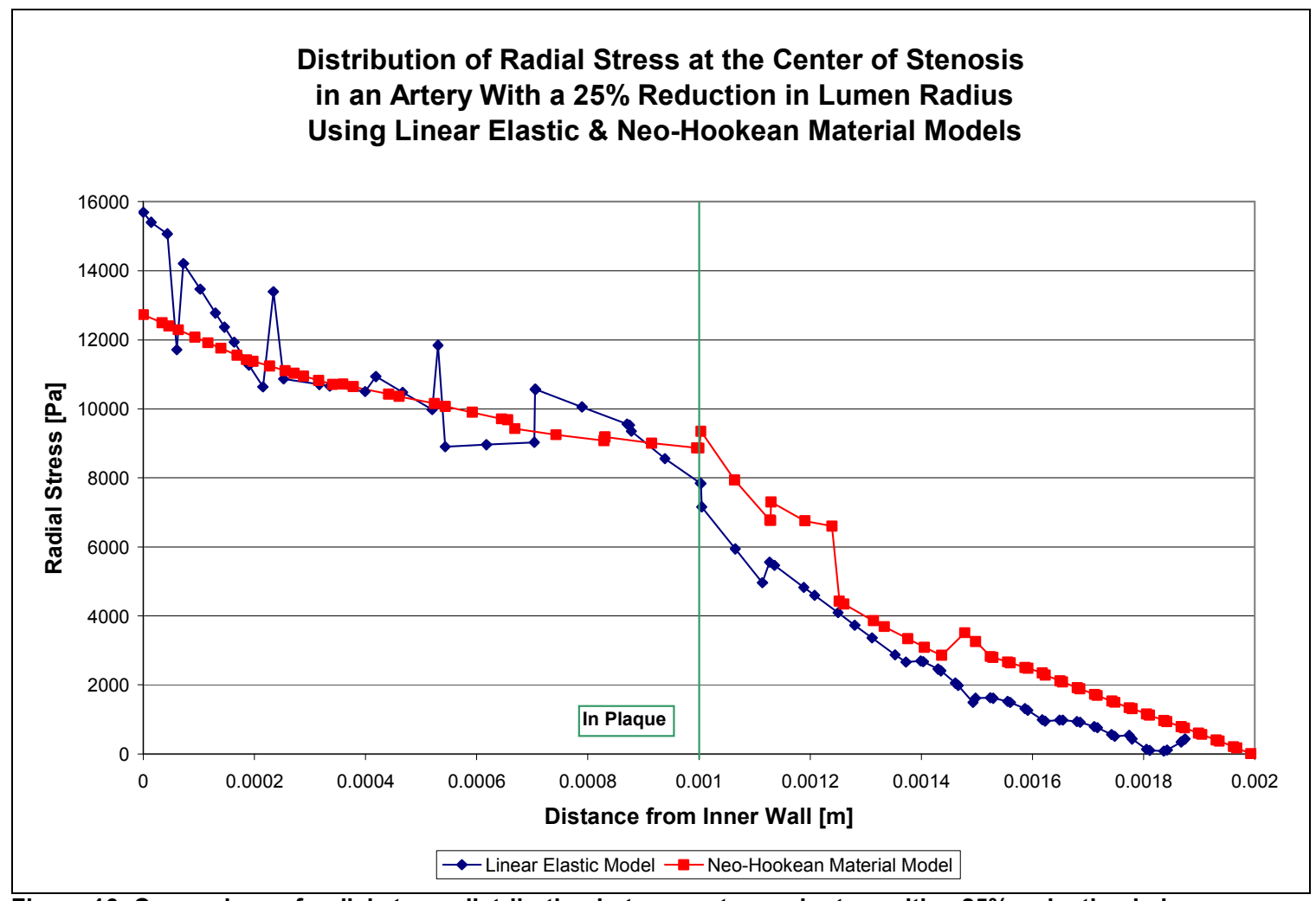

Figure 16: Comparison of radial stress distribution between a tapered artery with a $25 \%$ reduction in lumen radius modeled with an isotropic linearly elastic material and an isotropic neo-Hookean material model. 


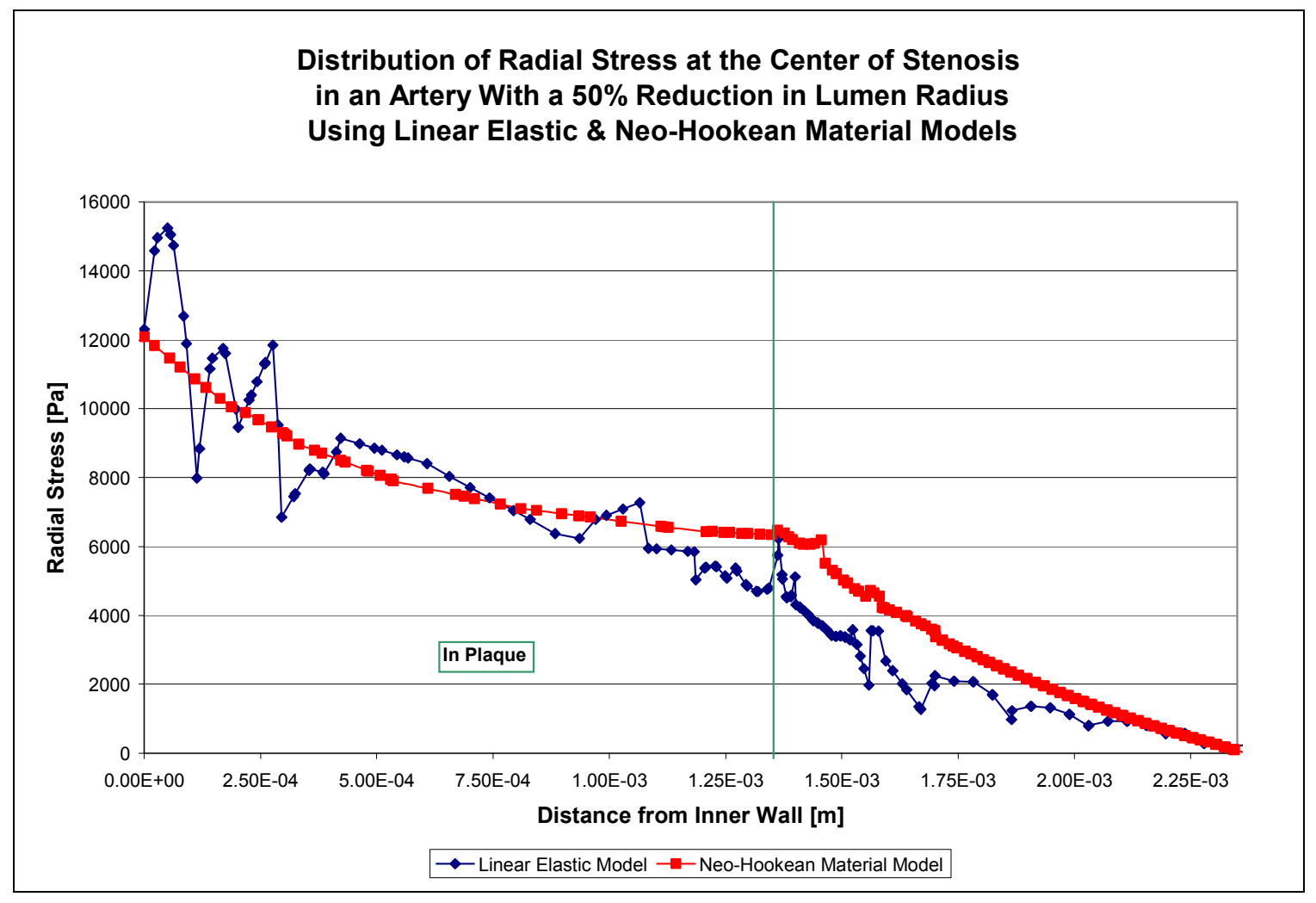

Figure 17: Comparison of radial stress distribution between a tapered artery with a $50 \%$ reduction in lumen radius modeled with an isotropic linearly elastic material and an isotropic neo-Hookean material model. 


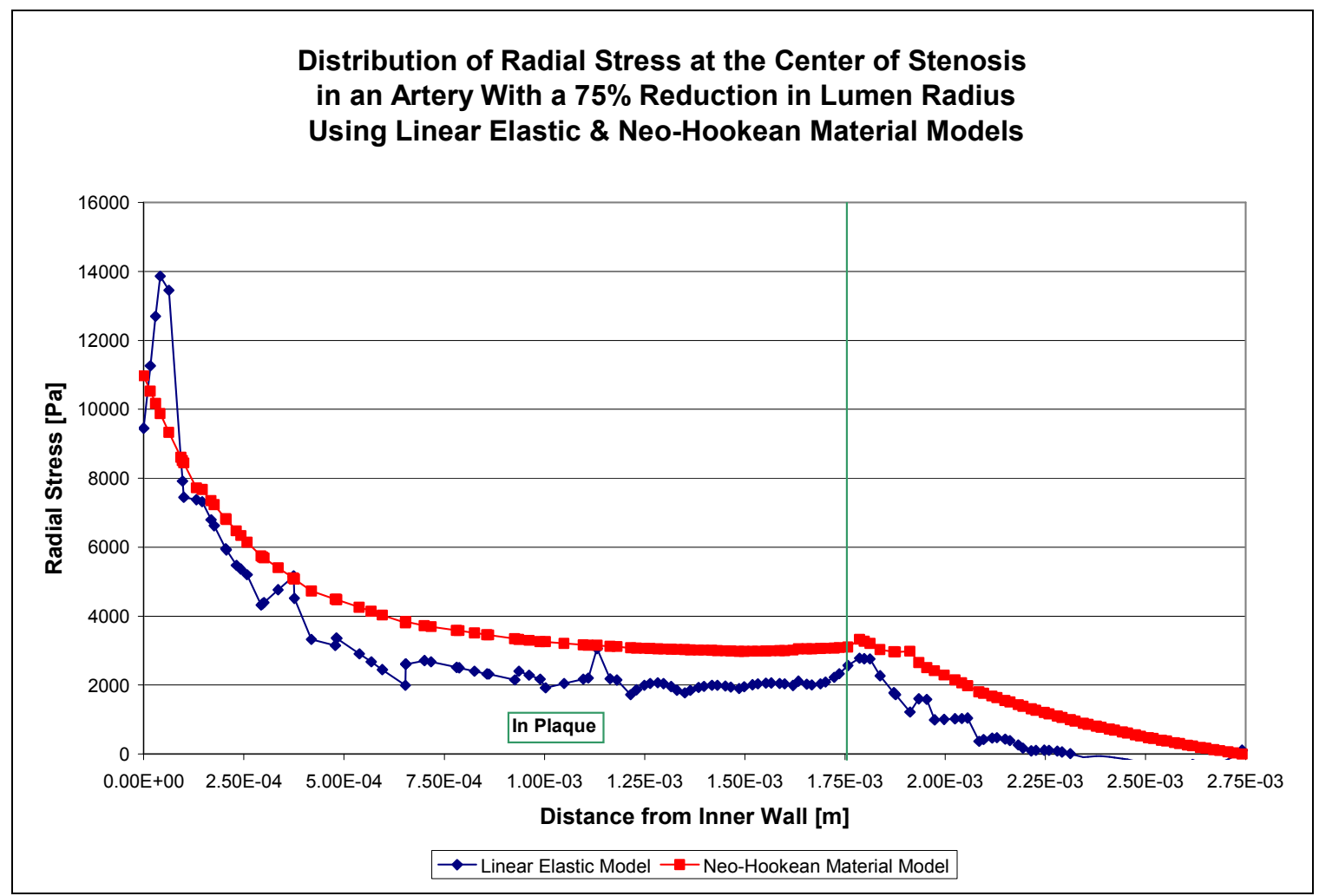

Figure 18: Comparison of radial stress distribution between a tapered artery with a $75 \%$ reduction in lumen radius modeled with an isotropic linearly elastic material and an isotropic neo-Hookean material model.

\section{E. Wall Shear Stress}

The pressure drops were used as described in Table 1 to produce the prescribed average flow rates. Figures 19 through 22 show the wall shear stress (WSS) distribution in each tapered model. As predicted by Equation 20, the shear stress rises rather dramatically as the lumen radius is reduced. Wall shear stresses in the healthy model reached a maximum of slightly over $3 \mathrm{~Pa}$ near the distal end. In the healthy portions of the 
models featuring stenosis, the WSS never exceeded about $4 \mathrm{~Pa}$. These values are in agreement with the previously discussed studies on WSS.

The minimally stenosed artery model only showed slightly elevated WSS; this is not surprising given that the reduction of the lumen radius was not very dramatic. The model featuring a $50 \%$ radius reduction saw a more profound increase in the WSS along the plaque compared to the milder case of the disease. Overall, as illustrated in Figure 23, the wall shear stress climbed to very dramatic levels at the center of stenosis as the amount of closing increased. The model featuring a $75 \%$ reduction in radius showed extremely high wall shear stresses, peaking to about 65 $\mathrm{Pa}$. This is greatly above the $35 \mathrm{~Pa}$ prediction to induce endothelial damage previously discussed. These values were computed using the more accurate "Laminar Flow" physics module, which was found to give somewhat lower predictions than the "Creep Flow" module as illustrated earlier. 


\section{Healthy Artery Wall Shear Stress}

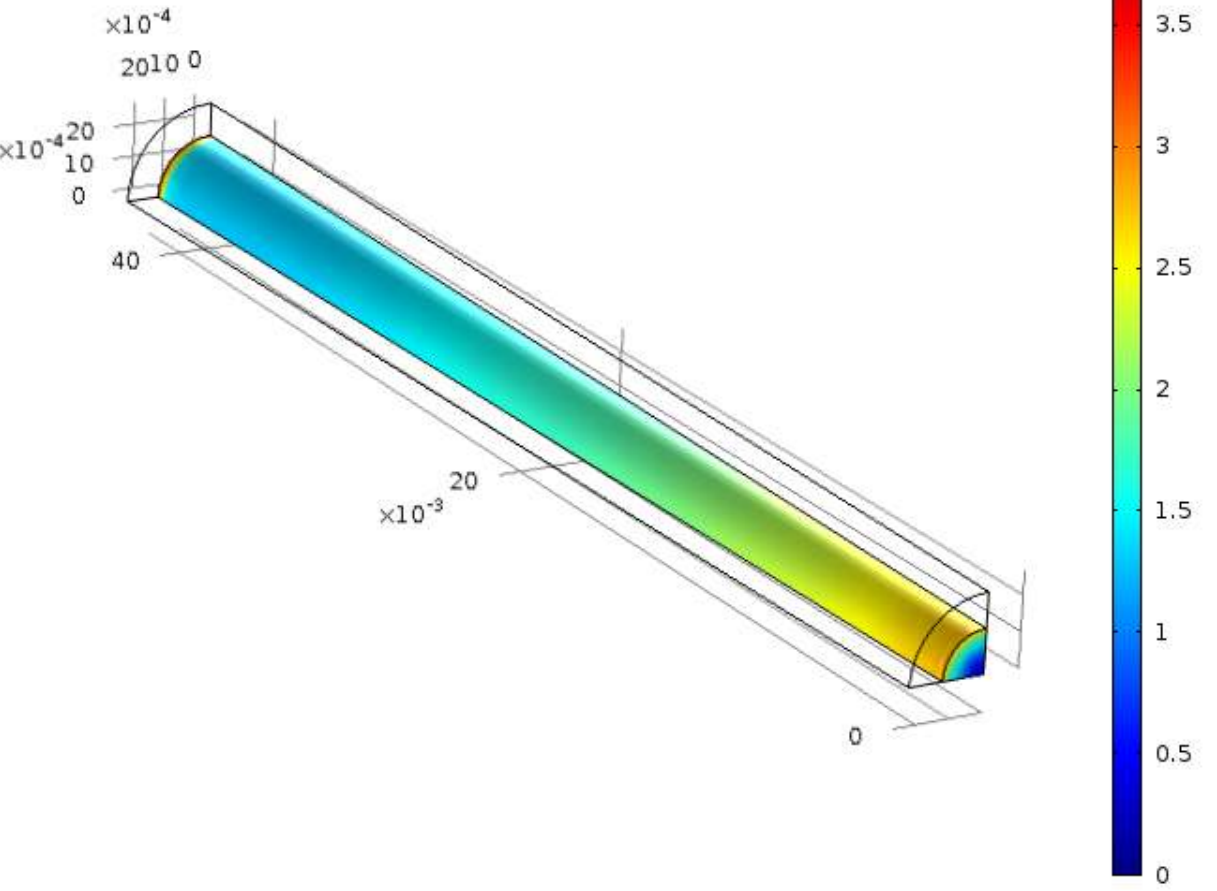

$4(\mathrm{~Pa})$

3.5

Figure 19: Distribution of wall shear stress in a model of a healthy tapered artery using creeping flow with an average volumetric flow rate. 


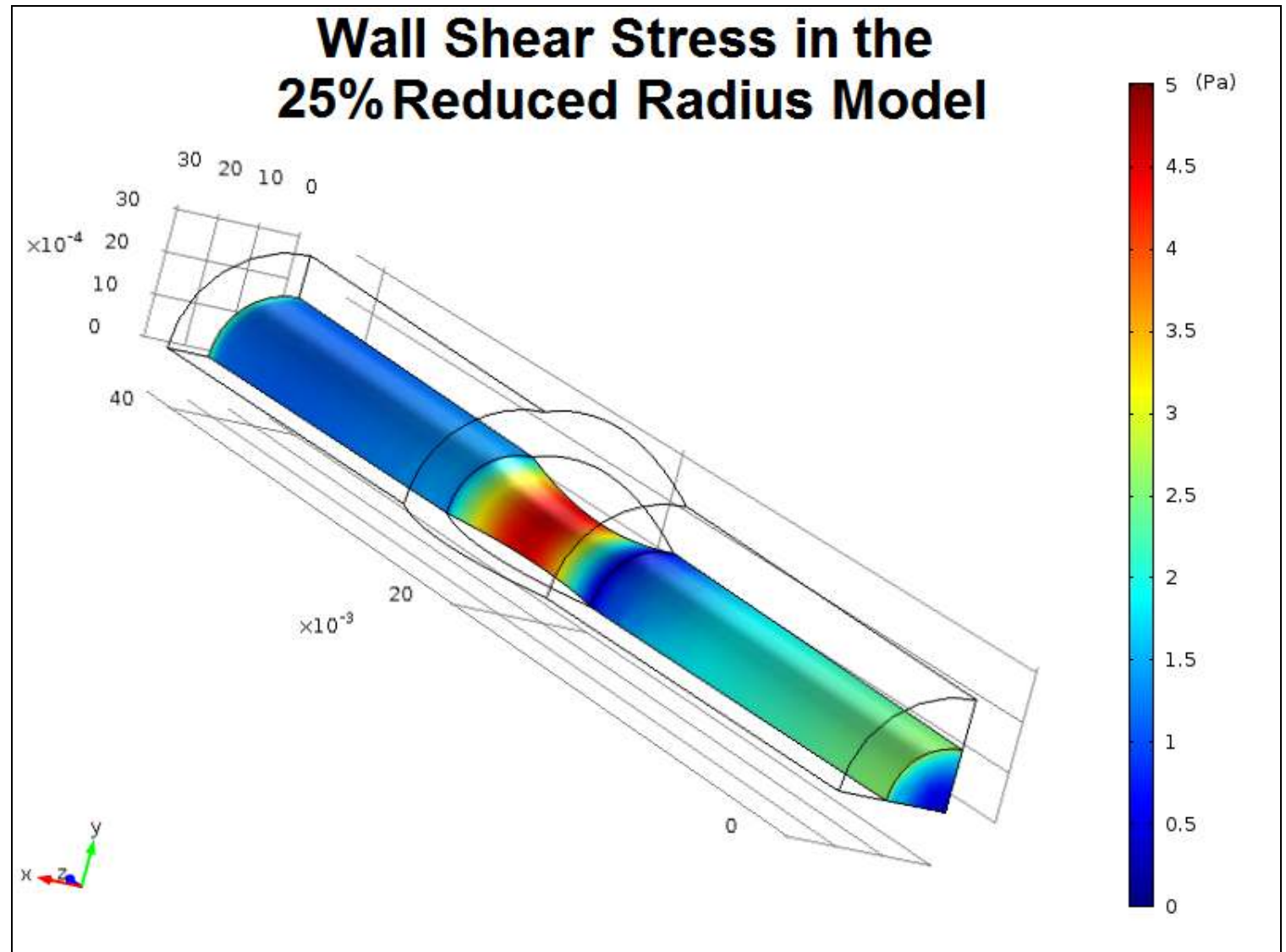

Figure 20: Distribution of wall shear stress in a model of a tapered artery with a $25 \%$ reduction in lumen radius using creeping flow with an average volumetric flow rate. 


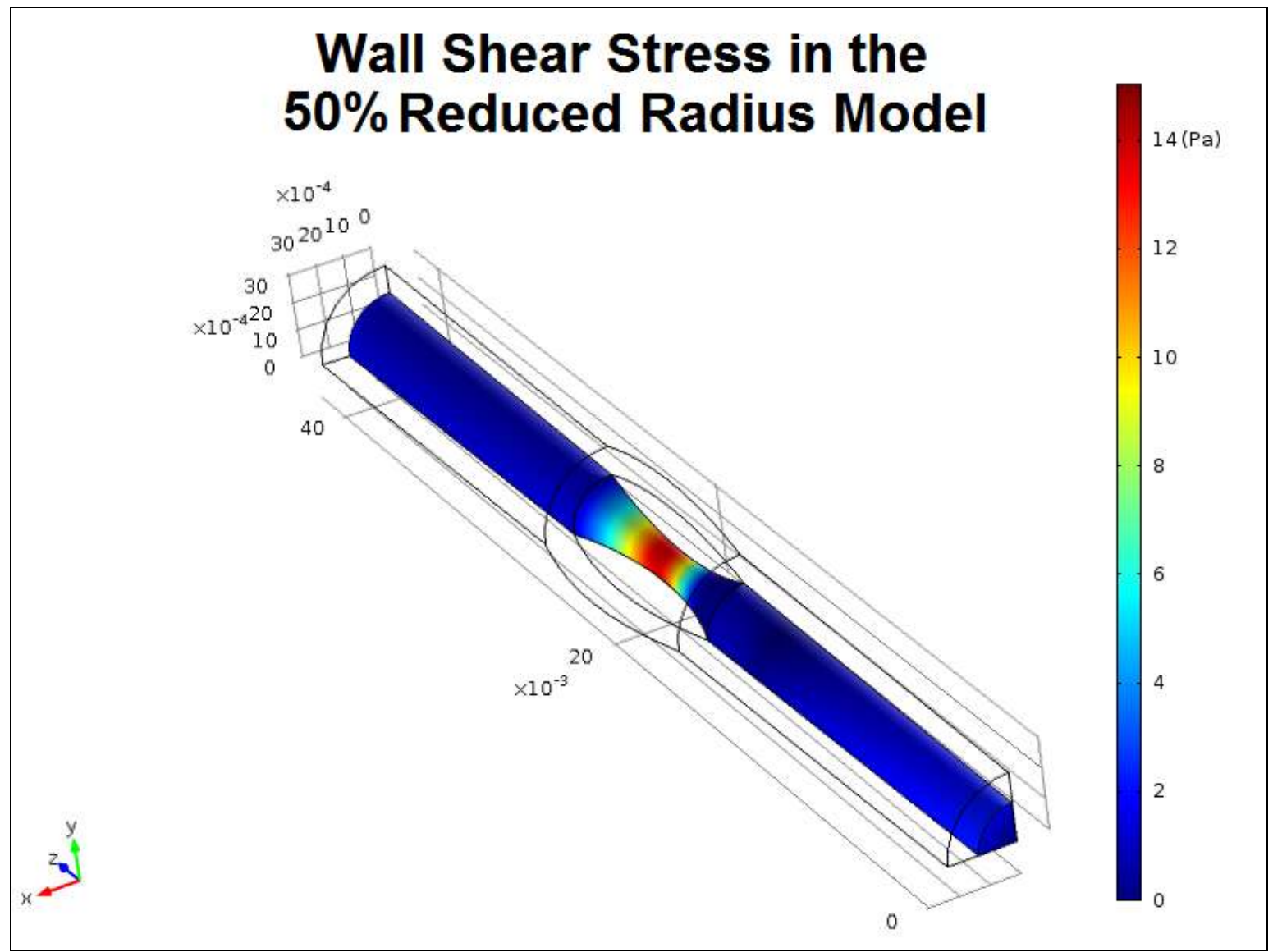

Figure 21: Distribution of wall shear stress in a model of a tapered artery with a $50 \%$ reduction in lumen radius using creeping flow with an average volumetric flow rate. 


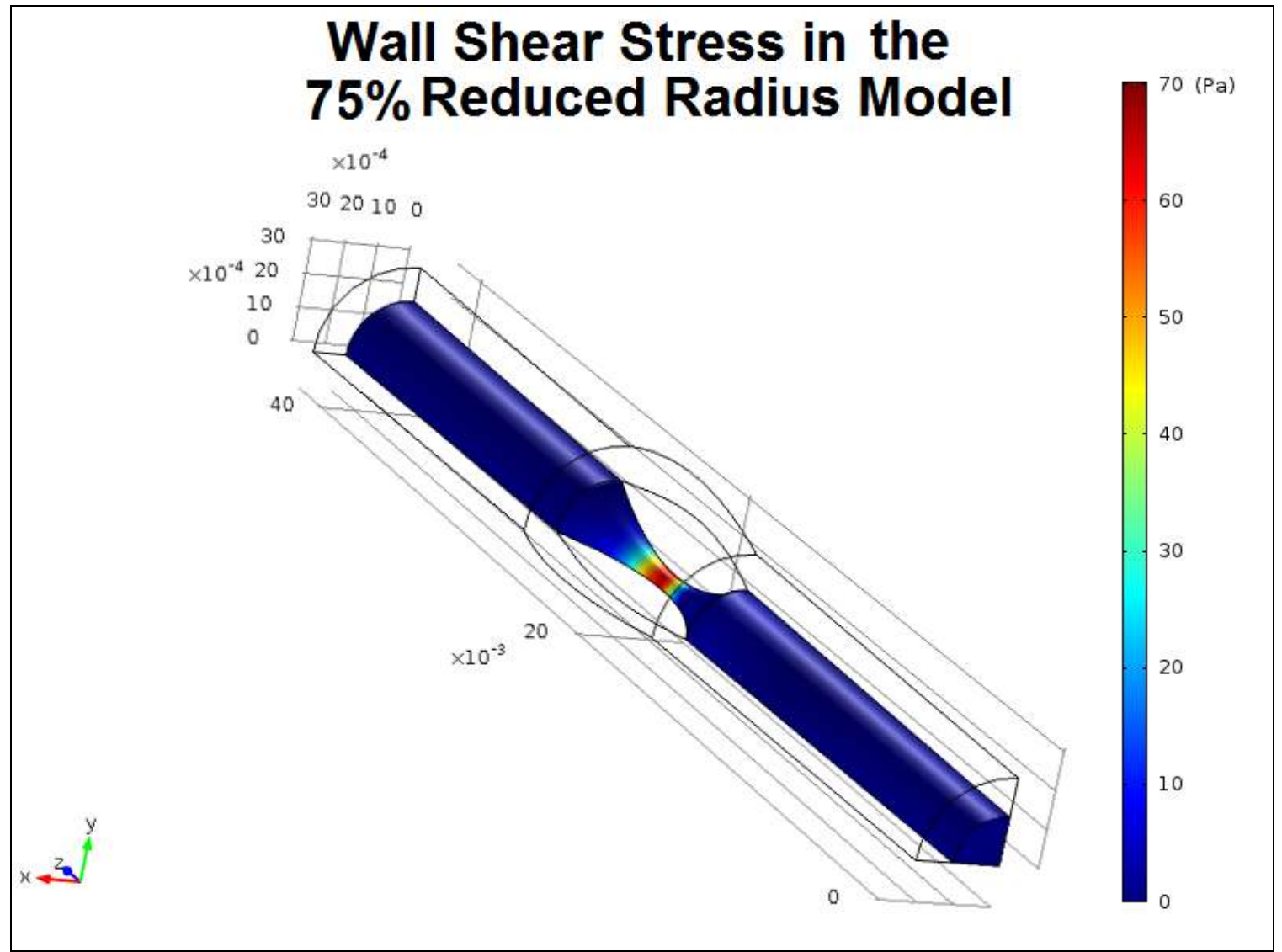

Figure 22: Distribution of wall shear stress in a model of a tapered artery with a $75 \%$ reduction in lumen radius using creeping flow with an average volumetric flow rate. 


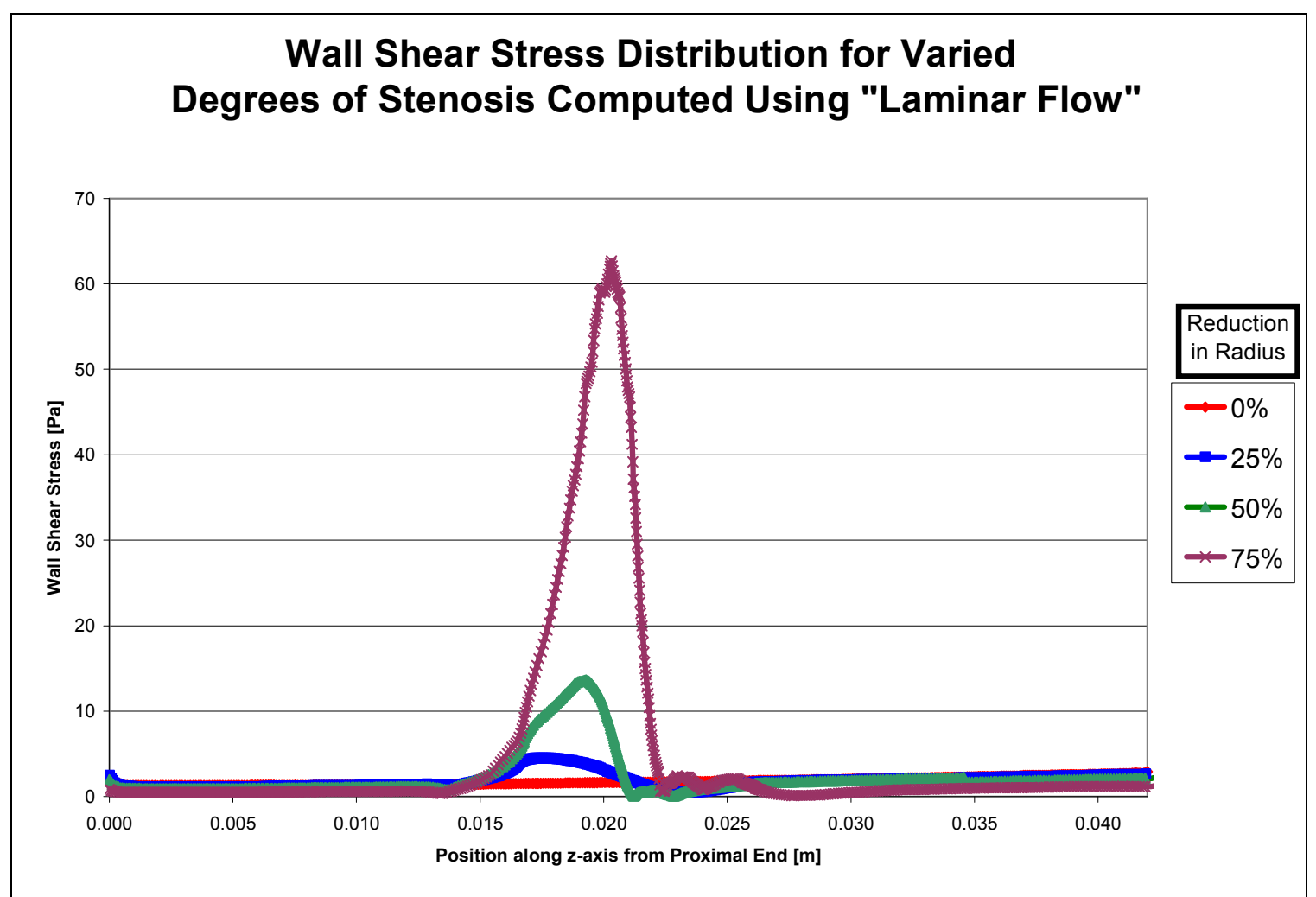

Figure 23: Comparison of the distribution of wall shear stress in each model of a tapered artery using creeping flow with an average volumetric flow rate.

\section{$\underline{\text { F. Time Varying Study }}$}

A series of time varying studies simulating one cardiac cycle were performed for each tapered model. First, the distension of the artery wall was examined. The displacements of three points on the inner wall along the length of the arties were tracked, with a point near the distal end, at the center of the artery length, and one near the proximal end as illustrated in

\section{Figure 24 .}




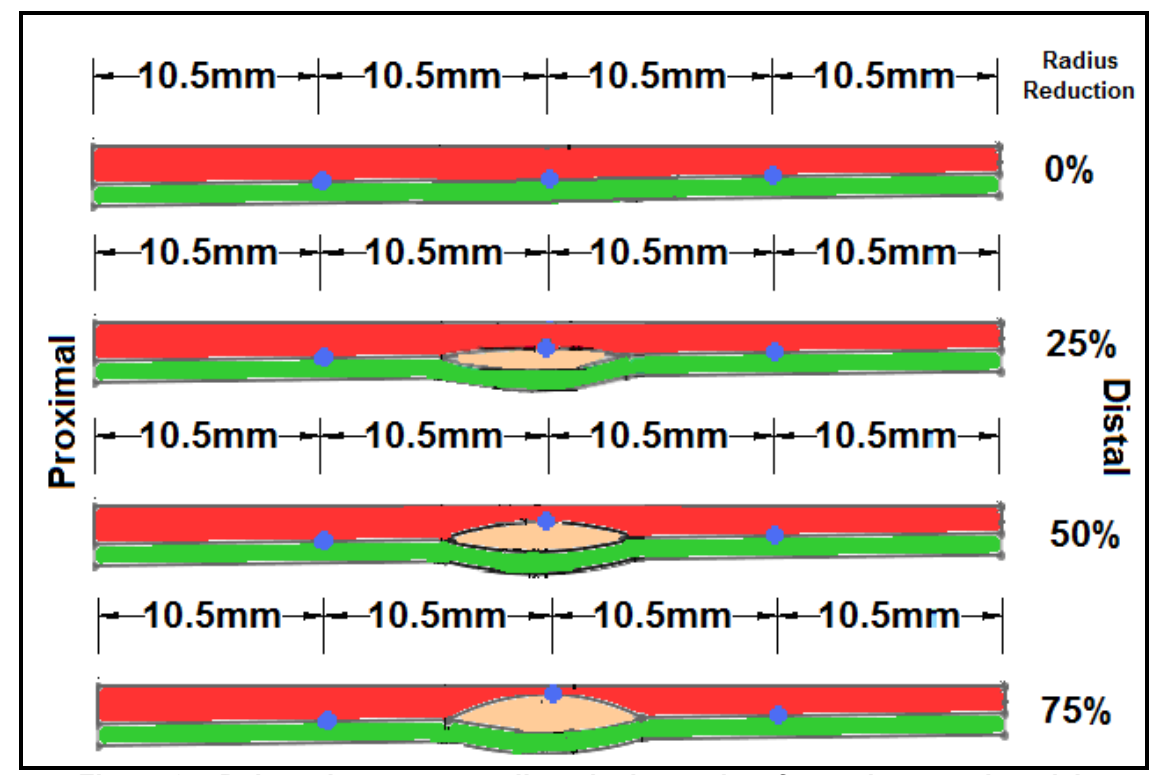

Figure 24: Points along artery wall tracked over time for each tapered model.

In Figure 25, the displacement over time of all three of these points is quite similar and corresponds well to the systemic blood pressure waveform (shown on the secondary axis) in the healthy artery model. The displacements are also correlated with the pressure and initial radius, which falls slightly from the proximal to distal end. It should be noted that the lumen radius at the peak pressure creates a change in area of about $10 \%$ compared to the low point, which consistent with observed in-vivo distension of the LAD. 


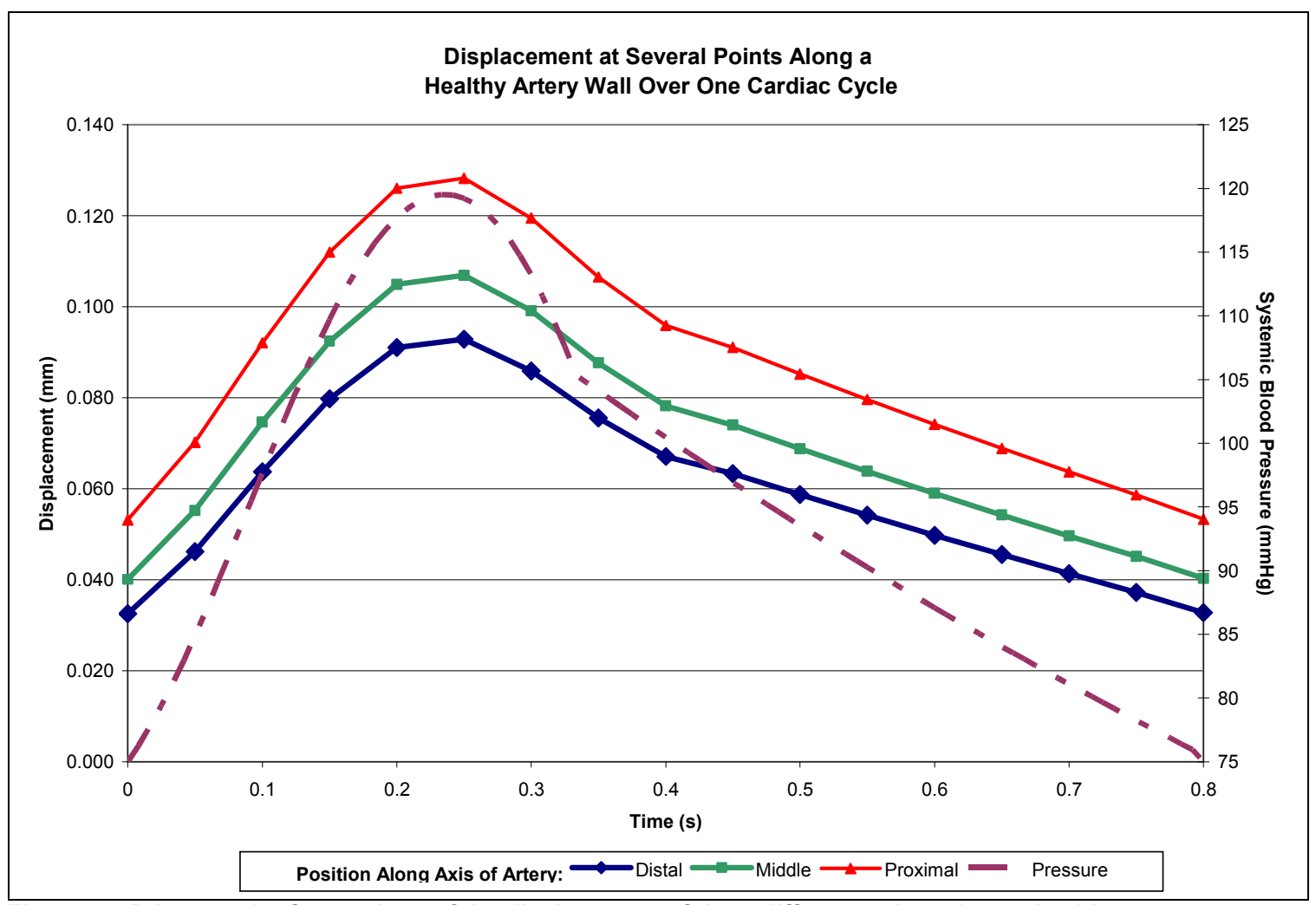

Figure 25: Primary axis-Comparison of the displacement of three different points along a healthy artery over one cardiac cycle; Secondary axis-Systemic blood pressure applying load.

Similar results were obtained for the distal and proximal points of the diseased arteries compared to the healthy model. The behavior of plaque differed between each case. For the $25 \%$ and $50 \%$ reduced radius models (Figure 26 and Figure 27), the change in radius between diastole and systole was roughly the same. The thicker of these two plaques appeared to experience greater displacement, but it is important to note that the initial radius of the stenosis is smaller. 


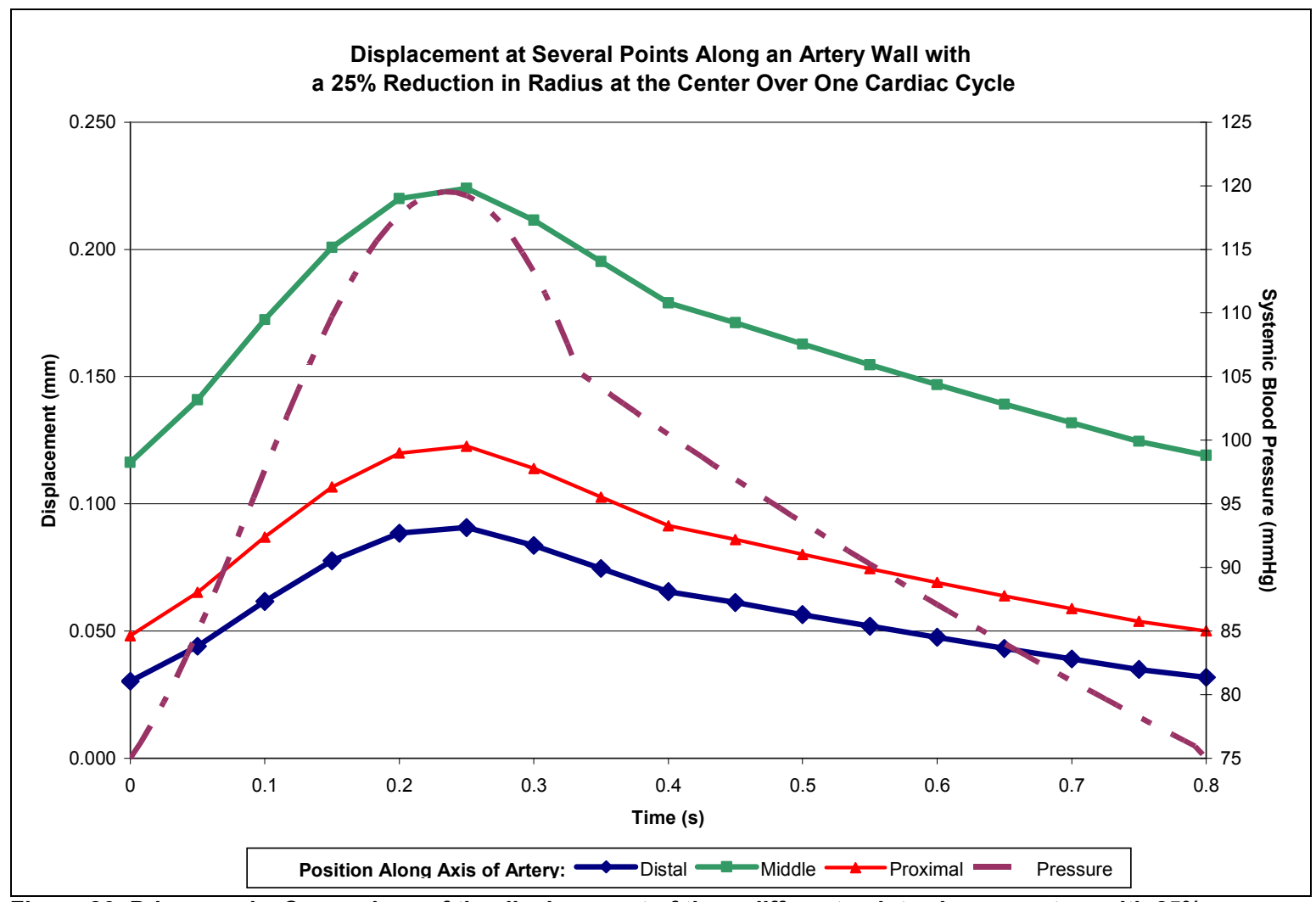

Figure 26: Primary axis-Comparison of the displacement of three different points along an artery with $25 \%$ reduced lumen radius at the center over one cardiac cycle; Secondary axis-Systemic blood pressure applying load. 


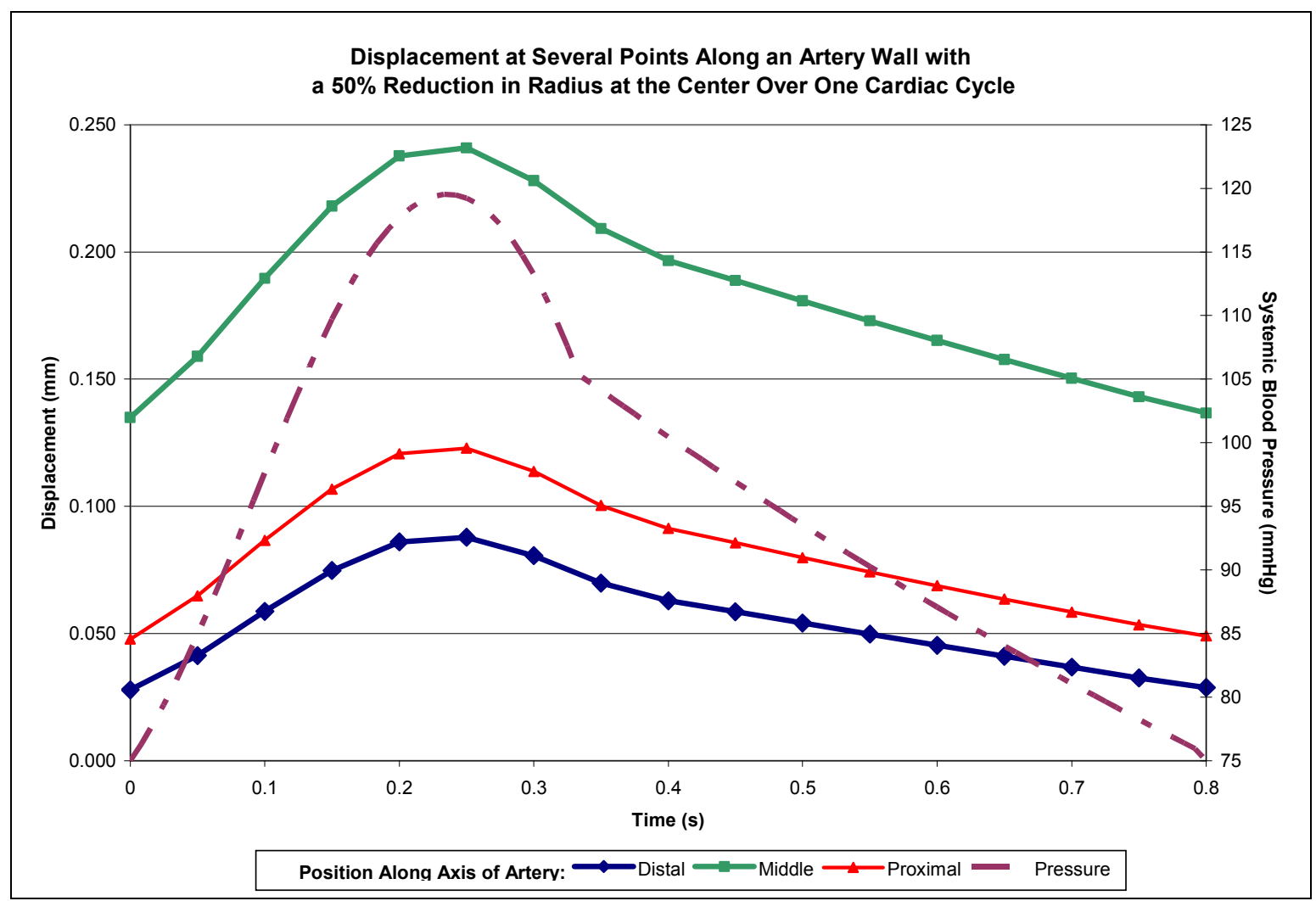

Figure 27: Primary axis-Comparison of the displacement of three different points along an artery with $\mathbf{5 0 \%}$ reduced lumen radius at the center over one cardiac cycle; Secondary axis-Systemic blood pressure applying load. 
In Figure 28, it can be seen that the largest plaque experienced very little movement over the cardiac cycle compared to the previous test cases. This demonstrates the reduced compliance associated with CAD; the difference between systole and diastole is diminished. The thick plaque makes a profound contribution to the mechanical behavior of this system.

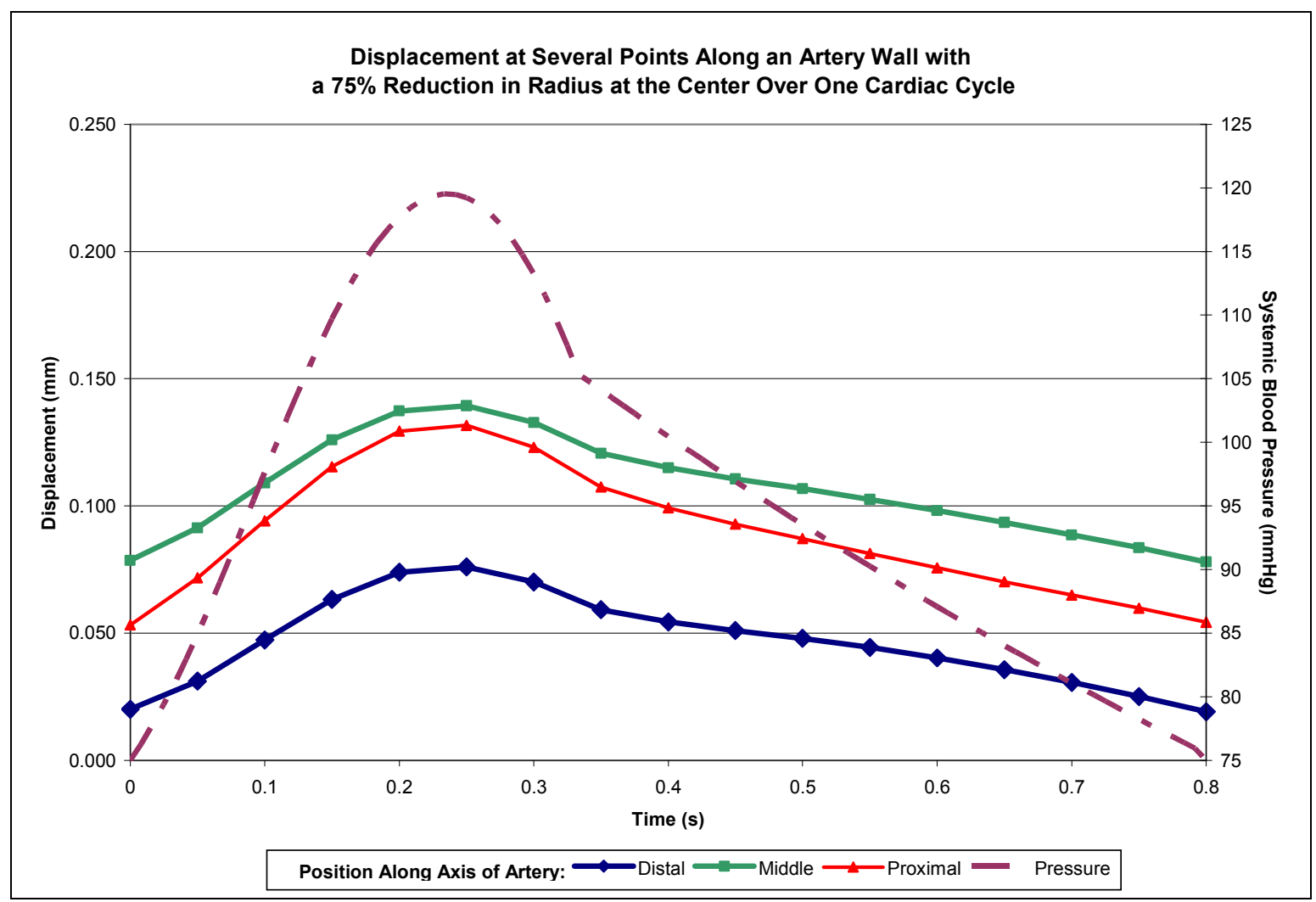

Figure 28: Primary axis-Comparison of the displacement of three different points along an artery with $75 \%$ reduced lumen radius at the center over one cardiac cycle; Secondary axis-Systemic blood pressure applying load. 
The wall shear stress was also followed over time at these same sampling points along the inner wall of each model. The pressure drop across the artery was modeled as a piecewise defined sinusoid that incorporated observed CFR data for mild and severe stenosis as well as the average flow rates specified in Table 1. Each pressure difference is shown on the secondary axis of Figures 29 through 32. For each model, the wall shear stress clearly follows the load curve, though there is a small phase difference that may be attributed to the initial conditions specified and the temporal resolution used by this simulation.

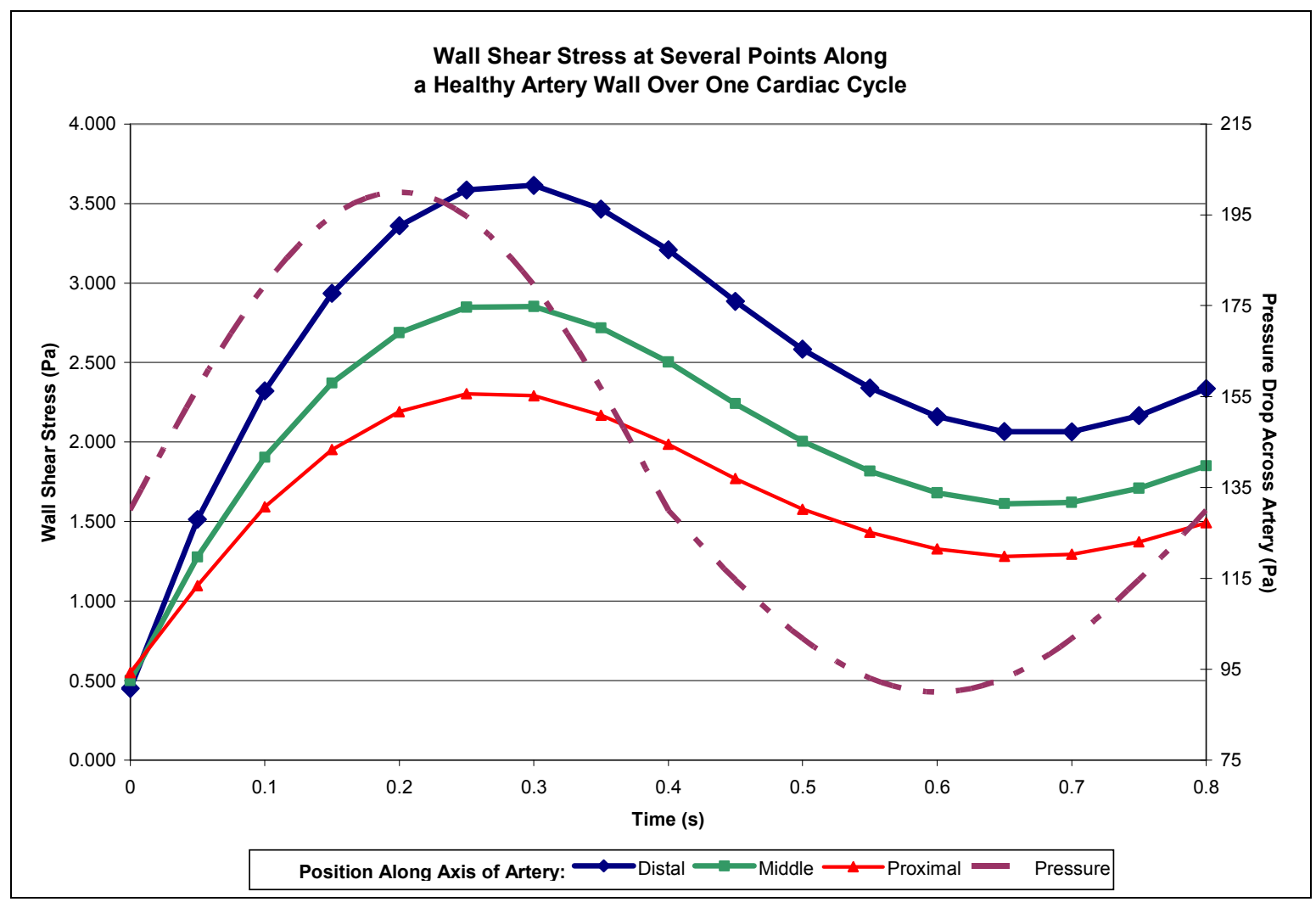

Figure 29: Primary axis-Comparison of the wall shear stress of three different points along a healthy artery over one cardiac cycle; Secondary axis-Systemic blood pressure applying load. 


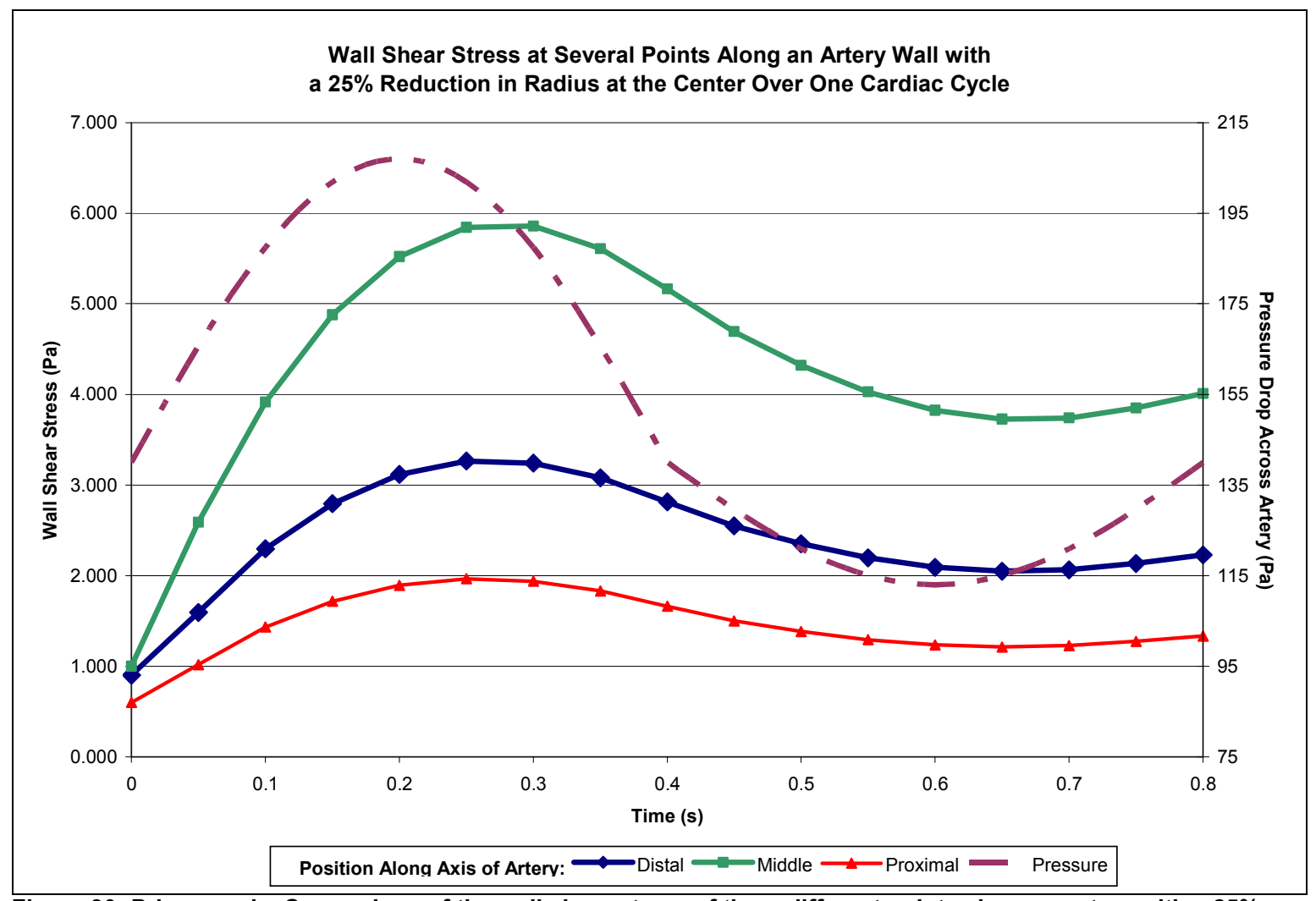

Figure 30: Primary axis-Comparison of the wall shear stress of three different points along an artery with a $25 \%$ reduced lumen radius over one cardiac cycle; Secondary axis-Systemic blood pressure applying load. 


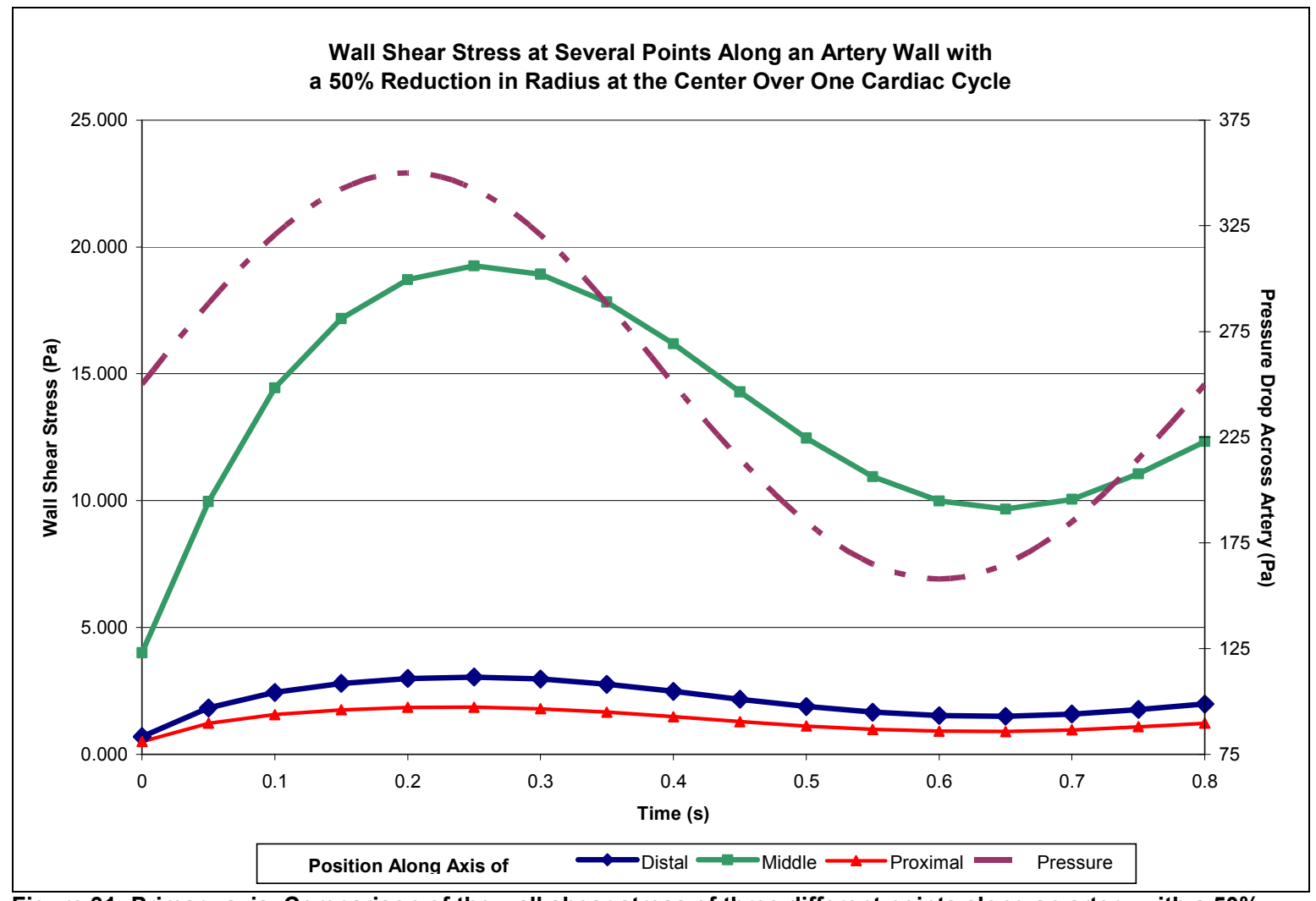

Figure 31: Primary axis-Comparison of the wall shear stress of three different points along an artery with a $50 \%$ reduced lumen radius over one cardiac cycle; Secondary axis-Systemic blood pressure applying load. 


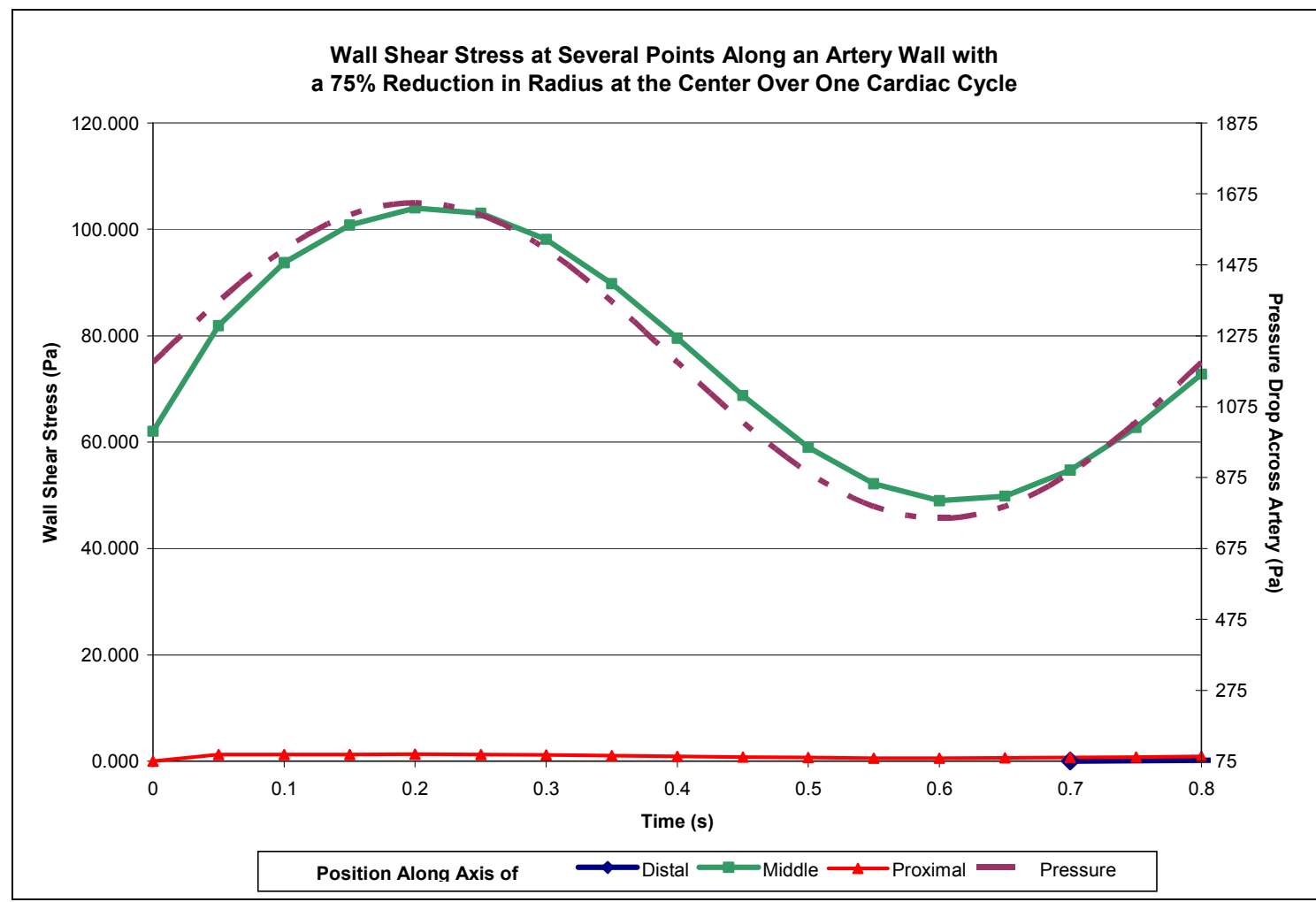

Figure 32: Primary axis-Comparison of the wall shear stress of three different points along an artery with a $75 \%$ reduced lumen radius over one cardiac cycle; Secondary axis-Systemic blood pressure applying load.

The magnitude of wall shear stress rises dramatically at the center as the amount of occlusion increases, as expected. These results are in line with these models earlier predictions for wall shear stress. 


\section{G. Summary and Discussion of Results}

Overall, these models performed as expected. The distension of the artery wall was near that reported in in-vivo studies. The range of wall shear stresses experienced by the model of a healthy LAD was also consistent with other previously mentioned studies. Wall shear stresses noticeably rose as the effective radius of the vessels decreased, also as expected.

The geometry used by these models has some minor limitations; the vessels feature a perfectly straight geometry and do not account for the presence of the smaller branches or arterioles. In addition, quarter symmetry was used to reduce computational costs, which precludes some more complicated geometries. There are some differences in the techniques used to collect these dimensions, which may account for some of the variability in dimensions and constants that were considered when creating these models. Ideally, a more realistic geometry could be obtained using a three dimension medical image, such as an MRI. Fortunately, it is readily possible to convert and import many file formats into COMSOL $\AA$, so this may be possible if the resources are made available. Such data could perhaps also be used to validate the models more thoroughly.

Another limitation of the model is that the geometry and mechanical properties of plaques can vary considerably in nature. Time constraints limited the values that could be tested as well. With greater 
resources, it would be possible to acquire mechanical properties from a single sample or set of experiments; this may help account for variability of measured properties, especially for more complicated material models.

As with any computational study, it would be ideal to use very high resolution meshes to ensure more consistent results. This is very possible with more powerful computers. In addition, more complicated constitutive relationships and material models may yield more accurate results. Unfortunately, these are also more computationally expensive. As with the previously discussed limitations, greater time and resources could be used to overcome some of said limitations. 


\section{Conclusions}

Several multiphysics models of a straight segment of the left anterior descending coronary artery were successfully created. This confirms our hypothesis that such models can readily be implemented in a commercially available software package such as COMSOL Multiphysics ${ }^{\circledR}$. The solid mechanical behavior was in line with the amount of distension that has been observed in-vivo for this artery. Predicted wall shear stress (WSS) for the healthy artery case was in agreement with literature. An increase in WSS was also seen as the amount of stenosis increased as expected.

With these models implemented, it can be easy to incorporate new components to the simulations. A more complex geometry or material model can be readily substituted. More importantly, it is possible to incorporate cardiovascular medical devices, such as stents, into models created in this software. Being able to accurately predict the behavior of such devices before prototypes are created can prove to be very useful and potentially cost saving. The multi-physics nature of these models allows many phenomena to be observed or incorporated, providing for more accurate simulations of coronary arteries and techniques used to treat their diseases. 


\section{REFERENCES}

1. Grigioni, e.a., Innovative Technologies for the Assessment of Cardiovascular Medical Devices: State-of-the-Art Techniques for Artificial Heart Valve Testing. Expert Review of Medical Devices, 2004.

2. McGarry, e.a., Analysis of the Mechanical Performance of a Cardiovascular Stent Design Based on Micromechanical Modelling. Computational Material Science, 2004.

3. Lanzer, e.a., The Need for Stent-Lesion Matching to Optimize Outcomes of Intracoronary Stent Implantation. Journal of Biomedical Materials Research Part B: Applied Biomaterials, 2013.

4. Burt, Drug-Eluting Stents: A Multidisciplinary Success Story. Advanced Drug Delivery Reviews, 2006.

5. Peng, e.a., Role of Polymers in Improving the Results of Stenting in Coronary Arteries. Biomaterials, 1996.

6. Elabbasi, B., Some Advances in Modeling Multiphysics-Biomedical Applications. Computational Fluid and Solid Mechanics, 2003.

7. Krstic, R., Human Microscopic Anatomy: An Atlas for Students of Medicine and Biology1991: Springer.

8. Lloyd-Jones D., A.R., Carnethon M., et al, American Heart Association Statistics Committee and Stroke Statistics Subcommittee Heart Disease and Stroke statistics--2009 Update: A Report from the American Heart Association Statistics Committee and Stroke Statistics Subcommittee. Circulation, 2009.

9. Coronary Artery Disease. MedlinePlus 2013 [cited 2013 May]; Available from: http://www.nlm.nih.gov/medlineplus/coronaryarterydisease.html.

10. White, R., Atherosclerosis and Arteriosclerosis: Human Pathology and Experimental Animal Methods and Models1989: CRC Press.

11. Bittl, Advances in Coronary Angioplasty. New England Journal of Medicine, 1996.

12. National Hospital Discharge Survey: 2009 table, Procedures by Selected Patient Characteristics - Number by Procedure Category and Age. CDC, 2009.

13. Guyton, H., Textbook of Medical Physiology1996: WB Saunders.

14. Snell, Clinical Anatomy By Regions. $9^{\text {th }}$ ed2011: Lippincott Williams \& Wilkins.

15. Kulkarni, Clinical Anatomy (A Problem Solving Approach). $2^{\text {nd }}$ ed2011: Jaypee Brothers Medical Publishers.

16. Lanzer, T., Pan Vascular Medicine: Integrated Clinical Management2002: Springer.

17. Shea, S.M., et al, Coronary Artery Imaging: 3D Segmented K-Space Data Acquisition with Multiple Breath Holds and Real Time Slab Following. Journal of Magnetic Resonance Imaging, 2001: p. 301-307.

18. Weber, O.M., Alastair J. Martin, and Charles B. Higgins, Whole Heart Steady State Free Precession Coronary Artery Magnetic Resonance Angiography. Magnetic Resonance in Medicine, 2003: p. 1223-1228. 
19. Van Geuns, R.J.M., et al, Volume Coronary Angiography Using Targeted Scans (VCATS): A New Strategy in MR Coronary Angiography. The International Journal of Cardiovascular Imaging 2001: p. 405-410.

20. Fayad, Z.A., et al, Noninvasive in-vivo Human Coronary Artery Lumen and Wall Imaging using Black-Blood Magnetic Resonance Imaging. Circulation, 2000: p. 506-510.

21. Gradus-Pizlo, I., et al, Left Anterior Descending Coronary Artery Wall Thickness Measured by High-Frequency Transthoracic and Epicardial Echocardiography Includes Adventitia. The American Journal of Cardiology, 2003: p. 27-32.

22. Perry R, J.M., Chew DP, Aylward PE, De Pasquale CG, Coronary Artery Wall Thickness of the Left Anterior Descending Artery Using High Resolution Transthoracic Echocardiography - Normal Range of Values. Echocardiography, 2013.

23. Perry, R., et al, Changes in Left Anterior Descending Coronary Artery Wall Thickness Detected by High Resolution Transthoracic Echocardiography. The American Journal of Cardiology 2008.

24. Kim, W.Y., et al, Three-Dimensional Black-Blood Cardiac Magnetic Resonance Coronary Vessel Wall Imaging Detects Positive Arterial Remodeling in Patients with Nonsignificant Coronary Artery Disease. Circulation, 2002.

25. Taylor, A.J., et al, Arterial Remodeling in the Left Coronary System: The Role of High-Density Lipoprotein Cholesterol. Journal of the American College of Cardiology 1999: p. 760-767.

26. Dodge, e.a., Lumen Diameter of Normal Human Coronary Arteries. Influence of Age, Sex, Anatomic Variation, and Left Ventricular Hypertrophy or Dilation. Circulation, 1992.

27. Nobusada Funabashi, e.a., Coronary Artery: Quantitative Evaluation of Normal Diameter Determined with Electron-Beam CT Compared with Cine Coronary Angiography - Initial Experience. Radiology, 2003.

28. Irmina Gradus-Pizlo, M.S.G.S., MD; Debbie Wright, MD; Douglas S Segar, MD; Harvey Feigenbaum, MD, Detection of Subclinical Coronary Atherosclerosis Using Two-Dimensional, High-Resolution Transthoracic Echocardiography. Journal of the American College of Cardiology, 2001. 37(5).

29. Weiss, e.a., Strain Measurement in Coronary Arteries Using Intravascular Ultrasound and Deformable Images. Transactions of the ASME, 2002.

30. Baldewsing, e.a., A Finite Element Model for Performing Intravascular Ultrasound Elastography of Human Atherosclerotic Coronary Arteries. Ultrasound in Medicine and Biology, 2004.

31. Maehara, e.a., Advances in Intravascular Imaging. Contemporary Reviews in Interventional Cardiology, 2009.

32. Li, Z., Stress Analysis of Carotid Plaque Rupture Based on in-vivo High Resolution MRI. Journal of Biomechanics, 2006.

33. Yabushita, e.a., Characterization of Human Atherosclerosis by Optical Coherence Tomography. Circulation, 2002.

34. Jang, e.a., In-vivo Characterization of Coronary Atherosclerotic Plaque by Use of Optical Coherence Tomography. Circulation, 2005. 
35. Lally, C.R., C. Prendergast P., Elastic Behavior of Porcine Coronary Artery

Tissue under Uniaxial and Equibiaxial Tension. Annals of Biomedical Engineering, 2004. 32(10).

36. Carlo Caiati, e.a., New Noninvasive Method for Coronary Flow Reserve Assessment: Contrast-Enhanced Transthoracic Second Harmonic Echo Doppler. Circulation, 1999.

37. Hozumi T., e.a., Noninvasive Assessment of Coronary Flow Velocity and Coronary Flow Velocity Reserve in the Left Anterior Descending Coronary Artery by Doppler Echocardiography: Comparison with Invasive Technique. Journal of the American College of Cardiology, 1998.

38. Edelman, e.a., Flow Velocity Quantification in Human Coronary Arteries with Fast, Breath-Hold MR Angiography. Journal of Magnetic Resonance Imaging 1993.

39. Kerner, D., Solving Windkessel Models with MLAB. 2007.

40. Herman, I., Physics of the Human Body2007: Springer.

41. Vito, R.D., S., Blood Vessel Constitutive Models-1995-2002. Annual Review of Biomedical Engineering, 2003.

42. Berge, Static Arterial Elasticity. Journal of Physiology, 1961.

43. Holzapfel, e.a., Determination of Layer-Specific Mechanical Properties of Human Coronary Arteries with Nonatherosclerotic Intimal Thickening and Related Constitutive Modeling Computational Biomechanics, 2005.

44. Broek, e.a., A Generic Constitutive Model for the Passive Porcine Coronary Artery. Biomechanics and Modeling in Mechanobiology, 2011.

45. Chen, H.e.a., The Layered Structure of Coronary Adventitia under Mechanical Load. Biophysical Journal, 2011. 101.

46. Karimi A., e.a., Measurement of the Uniaxial Mechanical Properties of Healthy and Atherosclerotic Human Coronary Arteries. Materials Science and Engineering, 2013.

47. Carew, e.a., Compressibility of the Arterial Wall. Circulation Research, 1968. 23.

48. Chuong C, F.Y., Compressibility and Constitutive Equation of Arterial Wall in Radial Compression Experiments. Journal of Biomechanics, 1984.

49. Holzapfel, e.a., A Layer-Specific Three-Dimensional Model for the Simulation of Balloon Angioplasty using Magnetic Resonance Imaging and Mechanical Testing. Biomedical Engineering Society, 2002.

50. Deng, e.a., New Experiments on Shear Modulus of Elasticity of Arteries. American Journal of Physiology, 1994.

51. Mengaldo G, e.a., A Comparative Study of Different Nonlinear Hyperelastic Isotropic Arterial Wall Models in Patient-Specific Vascular Flow Simulations in the Aortic Arch. MOX, 2012.

52. Lu, X.e.a., Shear Modulus of Porcine Coronary Artery: Contributions of Media and Adventitia. American Journal of Physiology: Heart and Circulatory Physiology, 2003.

53. Couade, e.a., Quantitative Assessment of Arterial Wall Biomechanical Properties Using Shear Wave Imaging. Ultrasound in Medicine and Biology, 2010.

54. Deale, C., Bubble Dynamics and Shock Waves2012: Springer. 
55. Lorenzini, C., Simplified Approach to Simulate 3D Haemodynamics in a Stenosed Artery. International Journal of Energy \& Technology, 2011.

56. Barrett, Experimental Measurement of the Mechanical Properties of Carotid Atherothrombotic Plaque Fibrous Cap. Journal of Biomechanics, 2009.

57. Tracqui, P.e.a., Mapping Elasticity Moduli of Atherosclerotic Plaque in-situ via Atomic Force Microscopy. Journal of Structural Biology, 2011.

58. Huang, e.a., The Impact of Calcification on the Biomechanical Stability of Atherosclerotic Plaques. Circulation, 2001.

59. Tang, e.a., Quantifying Effects of Plaque Structure and Material Properties on Stress Distributions in Human Atherosclerotic Plaques Using 3D FSI Models. Journal of Biomechanical Engineering, 2005.

60. Rahdert, D., Measurement of Density and Calcium in Human Atherosclerotic Plaque and Implications for Arterial Brachytherapy. Cardiovascular Radiation Medicine, 1999.

61. Berger, G., Lewis, Introduction to Bioengineering2000: Oxford University Press, USA.

62. Davies, P., Hemodynamic Shear Stress and the Endothelium in Cardiovascular Pathophysiology. Nature Reviews: Cardiology, 2009.

63. Lowe, G., et al., Blood Viscosity and Risk of Cardiovascular Events: the Edinburgh Artery Study. British Journal of Haematology 1997.

64. Pooler, C., Porth Pathophysiology: Concepts of Altered Health States2009: Lippincott Williams \& Wilkins.

65. Sircar, Principles of Medical Physiology2008: Thieme. 249.

66. Soulis, e.a., Wall Shear Stress in Normal Left Coronary Artery Tree. Journal of Biomechanics, 2005.

67. Sengupta, e.a., Image-Based Modeling of Hemodynamics in Coronary Artery Aneurysms Caused by Kawasaki Disease. Biomechanics and Modeling in Mechanobiology, 2012.

68. Yoshihisa Kurachi, A.T., Michael V. Cohen, Heart Physiology and Pathophysiology2000: Academic Press.

69. Souli, B., Arbitrary Lagrangian Eulerian and Fluid-Structure Interaction: Numerical Simulation2010: Wiley.

70. Jeremias, e.a., Coronary Artery Compliance and Adaptive Vessel Remodelling in Patients with Stable and Unstable Coronary Artery Disease. Heart, 2000.

71. Alfonso, e.a., Determinants of Coronary Compliance in Patients with Coronary Artery Disease: an Intravascular Ultrasound Study. Journal of the American College of Cardiology, 1994.

72. Junbo, e.a., Intravascular Ultrasound Imaging of Angiographically Normal Coronary Arteries: A Prospective Study in-vivo. British Heart Journal, 1994. 